\title{
Nonreciprocal interactions induced by water in confinement
}

\author{
Felipe Jiménez-Ángeles $\odot,{ }^{1}$ Katherine J. Harmon $\odot,{ }^{2}$ Trung Dac Nguyen $\odot,{ }^{3}$ \\ Paul Fenter $\odot,{ }^{4, *}$ and Monica Olvera de la Cruz $\circledast^{1,2,3,5,6, \dagger}$ \\ ${ }^{1}$ Department of Materials Science and Engineering, Northwestern University, Evanston, Illinois 60208, USA \\ ${ }^{2}$ Applied Physics Graduate Program, Northwestern University, Evanston, Illinois 60208, USA \\ ${ }^{3}$ Department of Chemical and Biological Engineering, Northwestern University, Evanston, Illinois 60208, USA \\ ${ }^{4}$ Chemical Sciences and Engineering Division, Argonne National Laboratory, Lemont, Illinois 60439, USA \\ ${ }^{5}$ Department of Physics and Astronomy, Northwestern University, Evanston, Illinois 60208, USA \\ ${ }^{6}$ Department of Chemistry, Northwestern University, Evanston, Illinois 60208, USA
}

(Received 26 June 2020; accepted 30 October 2020; published 17 November 2020)

\begin{abstract}
Water mediates electrostatic interactions via the orientation of its dipoles around ions, molecules, and interfaces. This induced water polarization consequently influences multiple phenomena. In particular, water polarization affects ion adsorption and transport, biomolecular self-assembly, and surface chemical reactions. Therefore, it is of paramount importance to understand water-mediated interactions modulated by nanoconfinement at the nanoscale. Here we investigate the effective interaction between two oppositely charged ions in different positions in water confined between two graphene surfaces. We find that the attraction between physisorbed ions is enhanced in the surface normal direction while the in-plane interaction is almost unaffected. The attraction in the surface normal direction is further enhanced by decreasing the confinement distance. Conversely, when one ion is intercalated into the graphene layers, the interaction becomes repulsive. Moreover, upon exchange of the ions' positions along the surface normal direction, the interaction energy changes by about $5 k_{B} T$. The nonequivalent and directional properties found here, referred to as nonreciprocal interactions, cannot be explain by current water permittivity models in confinement. Our x-ray reflectivity experiments of the water structure near a graphene surface support our molecular dynamics simulation results. Our work shows that the water structure is not enough to infer electrostatic interactions near interfaces.
\end{abstract}

DOI: 10.1103/PhysRevResearch.2.043244

\section{INTRODUCTION}

Water and ions, two basic components of living organisms and ubiquitous in numerous natural and technological processes, are strongly interconnected [1-3]. Water mediates interactions among ions, molecules, macromolecules, and interfaces through the polarization of its electric dipole moment. At the same time, charged species induce polarization of other molecules, surfaces, and the medium itself [4]. This leads to complex interactions, the details of which are not yet fully understood. In particular, the capability of a medium to be polarized and the resulting attenuation of electrostatic forces are typically quantified by the dielectric permittivity. This dielectric permittivity normalized by that of the vacuum $\left(\varepsilon_{0}\right)$, called the relative permittivity $\left(\varepsilon_{r}\right)$, is frequently assumed to be a distinctive property of a material and is often termed the dielectric constant. Such a denomination, however, is not applicable in nanomaterials, and especially for water in

\footnotetext{
*fenter@anl.gov

†m-olvera@northwestern.edu
}

Published by the American Physical Society under the terms of the Creative Commons Attribution 4.0 International license. Further distribution of this work must maintain attribution to the author(s) and the published article's title, journal citation, and DOI. confinement and near interfaces where the properties differ from the bulk [5-8].

The arrangement of water molecules at interfaces confers distinctive properties of interfacial water from those in the bulk [9]. For example, the varying water structure as a function of the distance to an interface modulates ionic interactions [10]. The ionic interactions near an interface are understood in terms of dielectric permittivity. Hence, considerable efforts have been made to determine the interfacial permittivity [11-16] of confined water. These studies have shown that the interfacial permittivity is anisotropic $[13,14]$ and is significantly diminished within $1 \mathrm{~nm}$ from an interface [12]. The connection between this interfacial dielectric permittivity and ionic interactions has been extensively studied using atomistic simulations and continuous models mainly to determine surface ionic profiles [17-19] and ion-specific effects [20,21]. Here we study ionic interactions between ions phsysiorbed and intercalated at the water-graphene interace. We show that a dielectric function, even if anisotropic effects due to confinement are included and nonpolarizable ions of equal size are considered, cannot describe the interactions between ions at interfaces (intercalated and physisorbed) and the broken symmetries when the ions are interchanged (see below).

The graphene-water interface is both technologically relevant and lacks the complexities of heterogeneous surfaces (e.g., proteins), making it an ideal system to investigate fundamental properties of ionic interactions in confinement. 
Graphene possesses extraordinary properties such as partial wetting transparency, electrical conductivity, and mechanical strength [22,23]. The graphene-water interface is of interest for water desalination [24,25], for electrochemical energy storage [26] and harvesting [27], as a trans-electrode membrane to characterize biomolecules [28], and in many other applications. Previous studies at air-water [29,30] and at graphene-water interfaces [19,31] have discussed ion adsorption (density profiles) and the effects from ions size, polarizability, chemical structure ( $\mathrm{such}$ as $\mathrm{OH}^{-}$and $\mathrm{H}_{3} \mathrm{O}^{+}$), and solvation energy, as well as the surface structure, among other factors. Here we investigate ion-ion effective interactions near the water-graphene interface. We consider intercalated and physisorbed nonpolarizable model ions of equal size but opposite charge. We show that near the interface the interactions between oppositely charged physisorbed ions are nonequivalent by interchanging the ions' positions with respect to the surface and can be shifted from repulsive to attractive by changing the ionic conformation, that is, when the position of one ion is changed from intercalated to physisorbed.

First, we investigate the water polarization near a graphene surface in the absence of any free charges and observe the changes of the interfacial water polarization in the presence of a nearby ion. Second, we compare the molecular interfacial water structure predicted by the simulation with that determined by x-ray reflectivity measurements. Third, we evaluate the effective ion-ion interactions near the graphene surface both in the plane and along the surface normal and assess the effect of increasing the confinement. We explain our results in terms of the interfacial water polarization rather than the dielectric constant. We demonstrate that the symmetry breaking of ion-ion interactions is present in both symmetric nonpolarizable ion models and in polarizable models of water, ions, and graphene. Finally, we analyze our results in the framework of the prevailing continuum theories of electrostatics at interfaces and we demonstrate that continuum theories fail to capture a fundamental breaking of symmetries of ion-ion effective interactions near interfaces.

\section{METHODS}

\section{A. Molecular simulation models}

To understand the water-mediated and confinement-altered interactions, we investigate ion-graphene and ion-ion interactions for a pair of nonpolarizable model test ions (monovalent anion and cation of equal size) placed between two uncharged nonpolarizable graphene surfaces [see Fig. 1(a)]. These assumptions allow us to show that the phenomena uncovered here are not attributed to specific properties of ions, such as size and polarizability. Our findings are further confirmed by considering polarizable models of ions, water, and graphene (see Appendix A). The ions are investigated in different configurations with respect to the graphene surface. The simulation box consists of two graphene surfaces separated by a water layer of thickness $L_{\mathrm{w}}$; each surface is formed by four graphene layers, $i=0, \ldots, 3$, with an interlayer separation of $0.358 \mathrm{~nm}$ and 1008 carbon atoms per layer. The simulation box size is $5.065 \mathrm{~nm} \times 5.104 \mathrm{~nm} \times L_{z}$, where the box length along the $z$ direction, $L_{z}$, is adjusted according to the number (a)

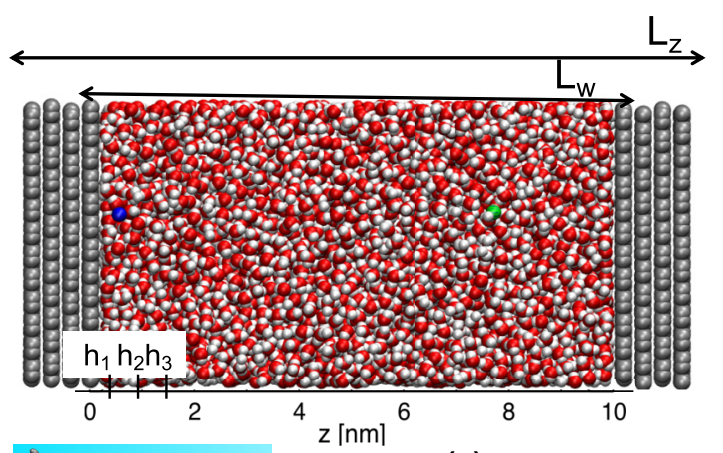

(b)

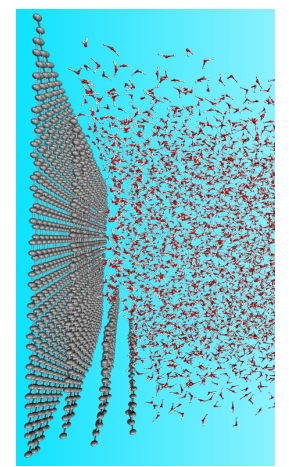

(c)

$$
\mathrm{G}_{0} \mathrm{G}_{1} \mathrm{G}_{2} \mathrm{G}_{3}
$$

FIG. 1. (a) Simulation setup consisting of a water layer of thickness $L_{\mathrm{w}}$ confined between two graphene surfaces. The response of the water polarization to electrical fields is investigated by placing two oppositely charged ions in different configurations. Here the cation (blue sphere) is at about $0.5 \mathrm{~nm}$ from the left graphene surface while the anion (green sphere) is at $z \approx 8 \mathrm{~nm}$; anions and cations have the same Lennard-Jones parameters $(\sigma=0.333 \mathrm{~nm}$ and $\epsilon=1.16 \times 10^{-2} \mathrm{~kJ} / \mathrm{mol}$ ) and differ only in their valence. $L_{\mathrm{z}}$ is the simulation box length in the $z$ direction, and $h_{i}$ with $i=1,2,3$ are three regions of $0.5 \mathrm{~nm}$ employed to quantify the water polarization next to the graphene surface. (b) Irregular surface employed for a direct comparison between x-ray reflectivity experiments and MD simulations; the surface is made of four partial graphene layers $\left(\mathrm{G}_{0}-\mathrm{G}_{3}\right)$ with the relative surface area of each graphene layer determined by the experimental XR results. (c) Calculation scheme employed to calculate the polarization field around an ion near the water-graphene interface.

of water molecules, $N_{\mathrm{w}} ; N_{\mathrm{w}}=8060$ and 2233 for $L_{\mathrm{w}}=9.8$ and $3 \mathrm{~nm}\left(L_{\mathrm{z}}=12.28\right.$ and $\left.5.63 \mathrm{~nm}\right)$, respectively. The water is simulated using the extended simple point charge $(\mathrm{SPC} / \mathrm{E})$ model [32], and the graphene parameters are taken from the all-atom optimized potentials for liquid simulations (OPLSAA) force field [33]. The ions' Lennard-Jones parameters are $\sigma=0.333 \mathrm{~nm}$ and $\epsilon=1.16 \times 10^{-2} \mathrm{~kJ} / \mathrm{mol}$ for both ions. Unless explicitly stated, the molecular dynamics (MD) simulations are performed using the three-dimensional periodic boundary conditions with slab correction (3DC) to mimic two-dimensional (2D) periodic boundary conditions in the $x$ and $y$ directions [34]. The system temperature is maintained at $T=298 \mathrm{~K}$ using a Nosé-Hoover thermostat.

To enable a one-to-one comparison between $\mathrm{x}$-ray reflectivity experiments and MD simulations, a separate model was built with no added ions in the system and with a graphene surface composed of four layers with different partial coverages 
[Fig. 1(b)]. This mimics the experimental surface wherein the graphene sample is made of multiple incomplete layers. The coverage of each layer was set to the value determined by the experimental x-ray reflectivity best-fit structure as follows: $G_{0}$ contained 1176 carbon atoms with $x \times y$ dimensions $5.157 \mathrm{~nm}$ $\times 5.955 \mathrm{~nm}$ (1 monolayer, ML); $G_{1}$ contained 924 carbon atoms, $x \times y=5.157 \mathrm{~nm} \times 4.679 \mathrm{~nm}(0.78 \mathrm{ML})$; $G_{2}$ contained 336 carbon atoms, $x \times y=5.157 \mathrm{~nm} \times 1.701 \mathrm{~nm}(0.29$ $\mathrm{ML})$; and $G_{3}$ contained 84 carbon atoms, $x \times y=5.157 \mathrm{~nm}$ $\times 0.425 \mathrm{~nm}(0.07 \mathrm{ML})$. These surface coverages are similar to those determined experimentally.

We analyze our results in terms of the polarization expressed as [35]

$$
\mathbf{P}(\mathbf{r})=\mathbf{P}_{1}(\mathbf{r})-\nabla \cdot \mathbf{P}_{2}(\mathbf{r})+\nabla \nabla: \mathbf{P}_{3}(\mathbf{r})+\cdots
$$

which includes the dipole moment per unit volume $\mathbf{P}_{1}$, the quadrupole moment $\mathbf{P}_{2}$, octupole moment $\mathbf{P}_{3}$, and all higher order moments. In water, the dipole moment is the main contribution to the polarization. Hence, it can be expressed as

$$
\mathbf{P}(\mathbf{r}) \approx \mathbf{P}_{1}(\mathbf{r})=\left\langle\sum_{i} \mu_{i} \delta\left(\mathbf{r}-\mathbf{r}_{i}\right)\right\rangle,
$$

where $\mu_{i}$ is the dipole moment of the $i$ th water molecule at $\mathbf{r}_{i}$, and \langle\rangle represents the ensemble average. The SPC/E water dipole moment is $\mu_{0} \equiv\left|\mu_{i}\right|=0.0489 e$ nm where $e$ is the positive elementary charge. The calculation scheme in molecular dynamics (MD) simulations is depicted in Fig. 1(c). Operationally, the instantaneous polarization is calculated as $\mathbf{p}(r, z)=\frac{\Delta \mathbf{m}}{\Delta V}$, where $\Delta \mathbf{m}=\sum_{i} \boldsymbol{\mu}_{i}$ includes the dipole moment $\left(\mu_{i}\right)$ of all the water molecules in the volume $\Delta V$ at $(r, z)$. The mean polarization is calculated as $\mathbf{P}(r, z)=$ $\langle\mathbf{p}(r, z)\rangle$, performing the ensemble average over at least $10^{4}$ independent configurations.

\section{B. Potential of mean force}

In an $N$-body system, the mean force exerted on the $i$ th particle is derived from the instantaneous forces from all the particles and is given by

$$
\mathbf{F}_{i}\left(\mathbf{r}^{n}\right)=-\nabla_{i} W^{(n)}\left(\mathbf{r}^{n}\right)=-\left\langle\nabla_{i} V\left(\mathbf{r}^{N}\right)\right\rangle^{(n+1)},
$$

where $W^{(n)}\left(\mathbf{r}^{n}\right)$ is the $n$-particle $(n \leqslant N)$ potential of the mean force $\left(\mathbf{F}_{i}\right), \nabla_{i}$ is with respect to the coordinates of the $i$ th particle $(i \leqslant n) ; V\left(\mathbf{r}^{N}\right)=\sum_{i<j}^{N} u\left(\mathbf{r}_{i j}\right)$ is the systems potential energy and $\mathbf{r}^{N}$ represents the $3 N$ particles' coordinates; $u\left(\mathbf{r}_{i j}\right)$ is the particles' pair interaction energy; and \langle\rangle$^{(n+1)}$ represents the ensemble average over $n+1 \ldots N$ particles. The potential of mean force is related to the $n$-particle probability distribution function $\mathscr{G}^{(n)}\left(\mathbf{r}^{n}\right)$ by

$$
W^{(n)}\left(\mathbf{r}^{n}\right)=-k_{B} T \ln \mathscr{G}^{(n)}\left(\mathbf{r}^{n}\right) .
$$

In our study, $n=2$ and particles 1 and 2 represent the two ions. The potential of mean force depends on the separation distance between the two graphene surfaces, $L_{\mathrm{w}}$, and the ions' positions with respect to the graphene surfaces, $\mathbf{r}_{2}$ and $\mathbf{r}_{1}$; $\mathbf{d}=\mathbf{r}_{2}-\mathbf{r}_{1}$ is the ions' relative position and $d=\left|\mathbf{r}_{2}-\mathbf{r}_{1}\right|$. We investigate the following cases:
(1) $W(z)=W\left(\mathbf{r}_{1}, \mathbf{r}_{2}\right)$ is the interaction of one ion, say 1, and the graphene surface along the surface normal direction, where $\left|\mathbf{r}_{1}\right|=z_{1} \lesssim 1.5 \mathrm{~nm},\left|\mathbf{r}_{2}\right| \rightarrow L_{\mathrm{w}} ; L_{\mathrm{w}} \approx 10 \mathrm{~nm}$. The interaction of ion 2 with the surface is calculated by exchanging the ions' position.

(2) $W_{\perp}(d)=W\left(\left|\mathbf{r}_{1}-\mathbf{r}_{2}\right|\right)$ is the ion-ion interaction along the graphene surface normal direction when $x_{1}=x_{2}, y_{1}=y_{2}$, and $z_{1}=0.28 \mathrm{~nm}$ and $z_{2} \lesssim 1.5 \mathrm{~nm}$, or by exchanging $z_{1}$ and $z_{2}$.

(3) $W_{\|}(d)=W\left(\left|\mathbf{r}_{1}-\mathbf{r}_{2}\right|\right)$ is the ion-ion interaction in the plane parallel to the graphene surface when $z_{1}=z_{2} \approx$ $0.28 \mathrm{~nm}$

(4) $W_{b}(d)=W\left(\left|\mathbf{r}_{1}-\mathbf{r}_{2}\right|\right)$ is the ion-ion interaction in bulk (i.e., without the graphene surfaces).

To calculate the potential of mean force (PMF), we use the umbrella sampling method [36], which is based on Eq. (4). The technique consists in performing biased sampling by fixing the ion of interest at designated positions along the reaction coordinate $\xi=z, d$. The test ion is fixed using a harmonic potential given as $u_{\text {umbrella }}(\xi)=-k\left(\xi-\bar{\xi}_{i}\right)^{2}$, where $\{\bar{\xi}, i=1, \ldots, M\}$ is a set of $M$ equilibrium positions and $k=$ $2000 \mathrm{~kJ} /\left(\mathrm{mol} \mathrm{nm}{ }^{2}\right)$ is the spring constant. The separation distance between two contiguous $\bar{\xi}_{i}$ and $\bar{\xi}_{i+1}$ equilibrium positions is $0.03 \mathrm{~nm}$, approximately. The system is simulated over at least $20 \mathrm{~ns}$ to generate a distribution functions around for each $\bar{\xi}_{i}$. The biased potential of mean force is related to the particle's distribution functions within the windows by Eq. (4). The unbiased PMF, the biased PMF, the biased probability distribution function, and the external potential are related in an exact way [37]. The weighted histogram analysis method (WHAM) is employed to construct the unbiased PMF [38].

\section{Continuum theory of electrostatics}

In the classical theory of electrostatics, an interface is modeled by taking into account two media of dielectric constants $\varepsilon_{1}$ and $\varepsilon_{2}$, respectively [35]. The dielectric mismatch between the two media leads to the following boundary conditions for an electric field $\mathbf{E}$ passing through the interface

$$
\begin{gathered}
\varepsilon_{1} E_{\perp}^{(1)}=\varepsilon_{2} E_{\perp}^{(2)}, \\
E_{\|}^{(1)}=E_{\|}^{(2)},
\end{gathered}
$$

where $E_{\perp}$ and $E_{||}$represent the electric field components perpendicular and parallel to the interface, respectively, passing through medium 1 or 2 as indicated by the superscripts. The boundary conditions in Eq. (5) imply that the work needed to bring a particle of charge $q$ from infinity to a distance $z$ from an interface of planar geometry is given by

$$
W_{\mathrm{c}}(z)=\frac{\alpha q^{2}}{4 \pi \varepsilon_{0} \varepsilon_{1} z},
$$

where the dielectric mismatch is quantified by $\alpha=\left(\varepsilon_{1}-\right.$ $\left.\varepsilon_{2}\right) / 2\left(\varepsilon_{1}+\varepsilon_{2}\right)[39,40]$.

\section{Molecular theory of permittivity}

In the molecular theory of dielectrics, the interfacial region is described by means of a local permittivity $\varepsilon$ [13], which is a function of the system's molecular parameters. The 
components of the permittivity tensor are calculated from the unperturbed interfacial water structure, i.e., in the absence of free charges within the interfacial region (see Appendix B). In a system with slab geometry [see Fig. 1(a)], the permittivity tensor is a diagonal matrix with components $\varepsilon_{\| \mid}(z) \equiv \varepsilon_{x x}(z)=$ $\varepsilon_{y y}(z)$, and $\varepsilon_{\perp}(z) \equiv \varepsilon_{z z}$,

$$
\varepsilon_{\|}(z)=1+\left(\frac{\beta}{2 V \varepsilon_{0}}\right)\left(\left\langle\mathbf{M}_{\|} \cdot \mathbf{p}_{\|}(z)\right\rangle-\left\langle\mathbf{M}_{\|}\right\rangle \cdot\left\langle\mathbf{p}_{\|}(z)\right\rangle\right),
$$

and for the inverse permittivity $\varepsilon_{\perp}^{-1}(z)$ in the direction perpendicular to the surface

$$
\varepsilon_{\perp}^{-1}(z)=1-\left(\frac{\beta}{V \varepsilon_{0}}\right) \frac{\left(\left\langle\mathbf{M}_{\perp} \cdot \mathbf{p}_{\perp}(z)\right\rangle-\left\langle\mathbf{M}_{\perp}\right\rangle \cdot\left\langle\mathbf{p}_{\perp}(z)\right\rangle\right)}{D_{\perp}}
$$

where $\|$ and $\perp$ indicate, respectively, the parallel and perpendicular components of the total dipole moment $\mathbf{M}$ and the instantaneous polarization $\mathbf{p}(z),\langle\rangle$ means the ensemble average, $\beta=1 /\left(k_{B} T\right), T$ is the absolute temperature, $k_{B}$ is the Boltzmann constant, $\varepsilon_{0}$ is the vacuum permittivity, and $V$ is the simulation box volume:

$$
D_{\perp}=1+\left(\frac{\beta}{V \varepsilon_{0}}\right) \int\left[\left\langle\mathbf{M}_{\perp} \cdot \mathbf{p}_{\perp}(z)\right\rangle-\left\langle\mathbf{M}_{\perp}\right\rangle \cdot\left\langle\mathbf{p}_{\perp}(z)\right\rangle\right] d V .
$$

Equations (7) and (8) are applicable in systems where three-dimensional (3D) periodic boundary conditions are assumed ( $x, y$, and $z$ directions). Ballenegger and Hansen [13] derived the expression for a two-dimensional (2D) periodic boundary ( $x$ and $y$ directions) where $D_{\perp}=1$. The results from simulations using $3 \mathrm{D}$ and $2 \mathrm{D}$ boundary conditions are quantitatively different.

\section{E. Experimental methods}

High-resolution $\mathrm{x}$-ray reflectivity (XR) experiments provide a sensitive probe of the molecular structure of welldefined interfaces via a direct measurement of the electron density distribution [41] and have been employed to study the interfacial water structure at numerous planar surfaces [42-45]. Such measurements then serve as an indirect probe of the molecular dipole orientation and polarization at the interface. Specular XR measurements of the graphene-water interface, i.e., along the graphene (0001) surface normal direction, were carried out using a $3 \times 10 \mathrm{~mm}$ sample of an epitaxial graphene (EG) thin film grown on a semi-insulating $6 \mathrm{H}-\mathrm{SiC}(0001)$ substrate $(\mathrm{EG} / \mathrm{SiC})$ immersed in ultrapure deionized water (DIW, nominal $\mathrm{pH}=7$ ). EG was grown by thermal decomposition of $\mathrm{SiC}$ under a partial pressure of $\mathrm{Ar}$ at $1500{ }^{\circ} \mathrm{C}$ according to methods previously described $[46,47]$. This approach produces incomplete graphene layers as a growth artifact. Nevertheless, the resulting sample with large-area graphene surfaces is better suited for XR measurements than mechanically exfoliated graphene, which produces micron-sized flakes. Measurements were performed at beamline 33-ID-D of the Advanced Photon Source at Argonne National Laboratory. A photon energy of $14 \mathrm{keV}(\lambda=$ $0.89 \AA$ ) was used, and the x-ray beam cross section measured approximately $\approx 50 \mu \mathrm{m} \times 1 \mathrm{~mm}(h \times w)$. The specular

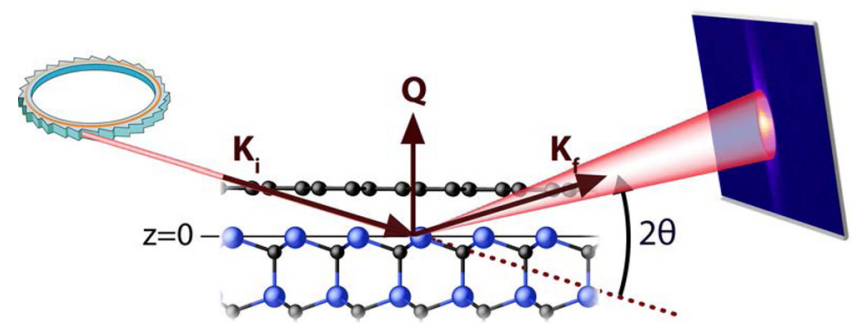

FIG. 2. The specular x-ray reflectivity (XR) is measured as a function of the perpendicular momentum transfer $Q=\left|\mathbf{K}_{f}-\mathbf{K}_{i}\right|$, where the incident $\mathbf{K}_{i}$ and reflected $\mathbf{K}_{f}$ X-ray beams vary by the scattering angle $2 \theta$.

XR data probe the time- and laterally-averaged (in the $x y$ plane) interface structure as a function of the vertical momentum transfer, $Q=4 \pi \sin (2 \theta / 2) / \lambda$, where $2 \theta$ is the x-ray scattering angle (see Fig. 2). The sample area probed by the $\mathrm{x}$-ray beam varies with the scattering angle and ranges from $\approx 1.58$ to $\approx 0.14 \mathrm{~mm}^{2}$ for the present measurements.

The XR best-fit structure was derived following a nonlinear least squares optimization procedure. In general, the electron density distribution of each atomic layer, $j$, along the substrate surface normal direction is modeled as a Gaussian with parameters to describe its position, $z_{j}$, coverage, $\theta_{j}$, and thermal widths, $u_{j}$. The total electron density profile is given by

$$
\rho(z)=\sum_{j} \frac{Z_{j} \Theta_{j}}{\sqrt{2 \pi u_{j}^{2}}} e^{\frac{-\left(z-z_{j}\right)^{2}}{2 u_{j}^{2}}}
$$

where $Z_{j}$ is the atomic number of the $j$ th layer. The reflectivity signal $R(Q)$ is related to the modulus squared of the Fourier transform of the electron density distribution and can be calculated for any set of model input parameters as

$$
R_{\text {calc }}(Q) \propto\left|\sum_{j} f_{0, j} \Theta_{j} e^{i Q z_{j}} e^{-Q^{2} u_{j}^{2} / 2}\right|^{2} .
$$

Here, the proportionality indicates that experimental considerations such as the angle-dependent transmission of $\mathrm{x}$ rays through the sample cell and surface roughness must be accounted for as extrinsic factors in the model (see Appendix C); $f_{0, j}$ is the atomic scattering factor of each element $Z_{j}$ [48] and accounts for the $Q$-dependent decay of the $x$-ray scattering intensity resulting from the spatial distribution of electrons around an atomic core. We evaluate the accuracy of the model via the goodness-of-fit metric $\chi^{2}$

$$
\chi^{2}=\frac{1}{N} \sum_{Q}\left(\frac{R_{\mathrm{calc}}(Q)-R_{\exp }(Q)}{\sigma(Q)}\right)^{2},
$$

where $N$ is the number of data points, $R_{\exp }(Q)$ is the experimentally measured reflectivity, and $\sigma(Q)$ is the experimental uncertainty at $Q$. The model parameters are refined until $\chi^{2}$ converges $\left(\chi^{2}=1\right.$ for a perfect fit within experimental uncertainties).

The model for the present system consists of a semi-infinite bulk $6 \mathrm{H}-\mathrm{SiC}(0001)$ substrate [49], one unit cell of $\mathrm{SiC}$ (i.e., six alternating $\mathrm{C}$-Si layers) to describe surface, up to eight $\mathrm{C}$ 
layers with the density of 2D graphene, and a semi-infinite layered water model described by Magnussen et al. [50]. The interfacial $\mathrm{SiC}$, graphene, and water structures are optimized according to Eqs. (10)-(12) while the bulk SiC structure is fixed.

Given a single adsorption surface (i.e., for a uniform, complete graphene layer), the layered water model includes a series of $m$ Gaussians ( $m=0,1,2 \ldots$ with the zeroth layer being closest to the adsorption surface) along the surface normal. The position $z_{m}$ and width $u_{m}$ of each layer are given by

$$
\begin{gathered}
z_{m}=z_{0}+m d_{w}, \\
u_{m}=\sqrt{u_{0}^{2}+m \bar{u}^{2}},
\end{gathered}
$$

where $z_{0}$ is the height of the zeroth Gaussian relative to the substrate surface (i.e., the interfacial water height), $d_{w}$ is the distance between adjacent Gaussian peaks, $u_{0}$ is the width of the zeroth layer, and $\bar{u}$ is the width broadening of subsequent layers such that the density asymptotically approaches that of bulk water $\left(\rho_{w}=330 e^{-} / \mathrm{nm}^{3}\right.$, where $e^{-}$is the negative elementary charge). We assume that the areal density of water in each Gaussian of this layered water distribution has the same coverage due to a lack of confinement in the lateral directions in our system. The coverage of each layer is then given by

$$
\Theta_{w}=\frac{A_{U C} d_{w}}{V_{w}},
$$

where $A_{U C}$ is the unit cell area of the $\mathrm{SiC}$ substrate and $V_{\mathrm{w}}=0.0299 \mathrm{~nm}^{3}$ is the effective volume of a water molecule in bulk assuming spherical symmetry.

As noted previously, the $\mathrm{EG} / \mathrm{SiC}$ growth methodology used in this work leads to partial layers of graphene. Therefore, we modified the layered water model [45] to incorporate multiple graphene surfaces, $G_{n}(n=0,1,2, \ldots)$, and assumed that water interacts in the same way with each graphene layer. Namely, above each exposed graphene surface exists the same intrinsic water structure according to Eqs. (14) and (15) but with a modulation due to the position $z_{n}$, width $u_{n}$, and coverage $\Theta_{n}$ of the adsorbing graphene layer. All together, the layered water structure is described by

$$
\begin{gathered}
z_{m, n}=z_{m}+z_{n}, \\
u_{m, n}=\sqrt{u_{m}^{2}+u_{n}^{2},} \\
\Theta_{w, n}=\Theta_{w} \frac{\Theta_{n}-\Theta_{n+1}}{\Theta_{\mathrm{ML}}},
\end{gathered}
$$

where the graphene monolayer coverage on $\mathrm{SiC}$ is $\Theta_{\mathrm{ML}}=$ 3.147 carbon atoms per $\mathrm{SiC}$ unit cell. Because the leastsquares fitting finds a local minimum in the parameter space, multiple structures consistent with the same XR data are possible. Therefore, we constrained the model parameters of the $\mathrm{SiC}$ and graphene surfaces based on previous chemically resolved measurements of the air/EG/SiC interface [44]. Further details of the XR analysis and all best-fit parameter values are reported in Appendix C.
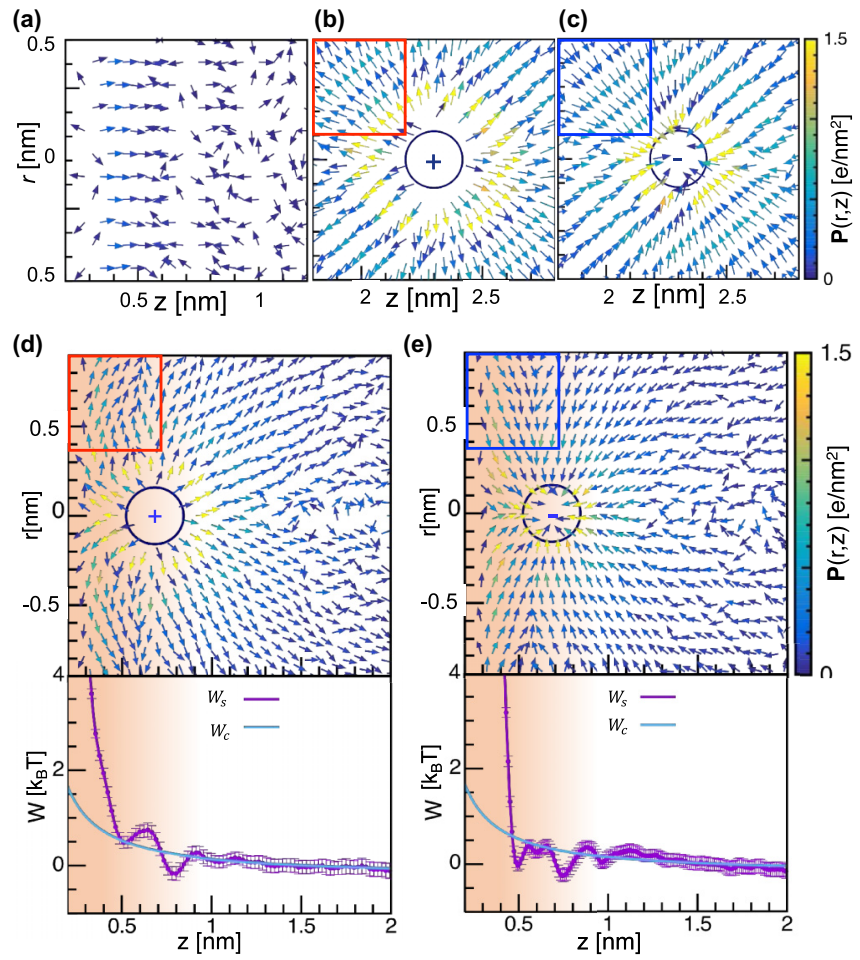

FIG. 3. Water polarization near the graphene surface and around ions. When both ions are away from each other and away from the graphene surface $\left(z_{1} \approx 2.3 \mathrm{~nm}\right.$ and $\left.z_{2} \approx 8 \mathrm{~nm}\right)$, an intrinsic polarization is observed (a) near the graphene surface, (b) around the cation, and (c) around the anion; the arrows indicate the polarization orientation and the magnitude is given by the scale bar at the right; $r$ is the distance from the ion center along the direction parallel the graphene surface $\left(\mathbf{e}_{\|}\right)$while $z$ is the distance from the graphene surface in the perpendicular direction $\left(\mathbf{e}_{\perp}\right)$. The water polarization near the graphene surface is changed by the presence of a nearby (d) cation or (e) anion. The bottom panels show the ion potential of mean force profile to bring an ion from the bulk to the graphene surface from our molecular dynamics simulations $W_{\mathrm{s}}$ (purple line) and calculated via Eq. (6) form the continuum theory of electrostatics $W_{\mathrm{c}}$ (light blue line). The boxes in panels (b) to (e) show the regions of the polarization maps that are different in bulk and in the presence of a physisorbed ion; red [in panels (b) and (d)] corresponds to the cation and blue [in panels (d) and (e)] corresponds to the anion.

\section{RESULTS}

\section{A. Interfacial water polarization}

The polarization field of water near the graphene surface and in the absence of nearby test charges is shown in Fig. 3(a). Even if the surface is uncharged and there are no nearby ions and no external electric field, the water is polarized perpendicularly to the graphene surface. This intrinsic interfacial polarization is due to the water dipole moment alignment caused by the solid surface and is also observed when polarizable models of water and graphene are used in the simulations (see Appendix A). This preferential orientation decays in an oscillatory way to a random polarization in the bulk $[z>1$ nm; see Fig. 3(a)]. As a reference for ions in bulk, we show the polarization field around a cation and around an anion far away $(z>2 \mathrm{~nm})$ from the interface in Figs. 3(b) and 3(c), 
respectively. In this case, the test charges are sufficiently far from the graphene surface and do not perturb the interfacial properties. At this separation distance from the graphene surface, the water polarization around the ions is spherically symmetric and decays without oscillations. However, the polarization field is not spherically symmetric when one ion is placed within $\approx 1 \mathrm{~nm}$ of the graphene surface [see Figs. 3(d) and 3(e)] for the nearby cation and anion, respectively) while the oppositely charged ion is kept away $(z \approx 8$ $\mathrm{nm})$. Moreover, the polarization response is asymmetric and unequal around the anion and cation. For example, the polarization of water around a cation in bulk is outward and radially symmetric whereas the component of the polarization in the $z$ direction is reversed in the presence of graphene [see the red boxes in Figs. 5(b) and 5(d)]. The polarization around an anion in the bulk is inward and radially symmetric whereas near a graphene surface the polarization is aligned parallel to the graphene surface [see the blue boxes in Figs. 5(c) and 5(e)]. This result contrasts with the continuum theory of electrostatic where the polarization fields for positive and negative test ions are assumed to have the same magnitude and opposite direction.

The unequal responses are quantified by the potential of mean force (PMF), $W(z)$, and are different for the anion and the cation (even if both have the same radius). For example, the cation's PMF at $z \approx 0.5 \mathrm{~nm}$ is approximately 0.5 $k_{B} T$ while the anion's PMF is approximately $0 k_{B} T$. Additionally, for $z \lesssim 0.5 \mathrm{~nm}$ the PMF becomes steeper for the anion than for the cation. The interfacial ion specificity has been attributed to the ionic polarizability, size, and valence. However, our results show that solely a change in sign gives rise to a pronounced ion specificity via the asymmetric water polarization response to the sign of the ions. In continuum electrostatic theory, the change in the dielectric permittivity at the interface is modeled by the image charge method, which from water to graphene would assign an equally repulsive force for the negative and positive ions [35] [see light blue line in Figs. 3(d) and 3(e)]. This symmetry is also obtained in continuum models for electrolytes in confinement [40,51,52].

\section{B. Interfacial water electron density distribution}

Here, we focus on the details of the water structure in the absence of ions and the relationship between XR experiments and the simulation results. Analysis of the XR data [red line in Fig. 4(a)] reveals a graphene-water electron density profile with four partial surfaces to which water adsorbs [Fig. 4(b)]. A comparison with XR data of graphene in air [blue line in Fig. 4(a)] reveals the sensitivity of the measurement to the water structure. The adsorbing surface includes three layers of graphene, $G_{1}, G_{2}$, and $G_{3}$ with fractional layer coverages of $0.84,0.31$, and $0.1 \mathrm{ML}$, respectively, and a reconstructed carbon buffer layer, $\mathrm{G}_{0}$, with complete coverage and which separates true $2 \mathrm{D}$ graphene $\left(\mathrm{G}_{1}-\mathrm{G}_{3}\right)$ from the $\mathrm{SiC}$ substrate below (see the complete interface structure in Appendix $\mathrm{C}$ and Table I for details).

In general, the XR measurements and MD simulations reveal qualitatively equivalent water distributions adsorbed on the partial graphene surfaces [Fig. 4(b)]. Both the XR-derived structure and the MD prediction show a weakly modulated

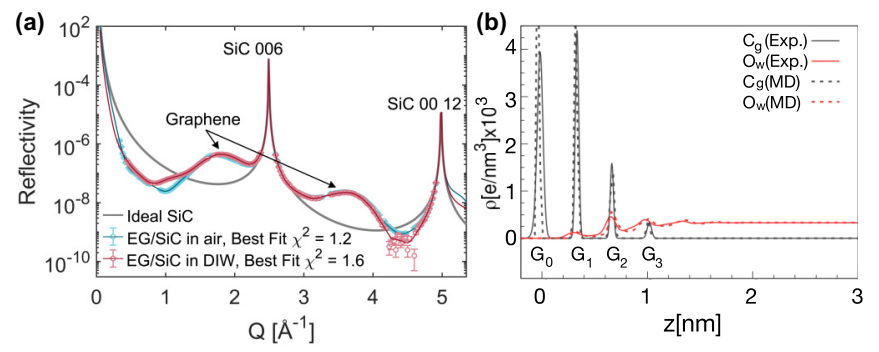

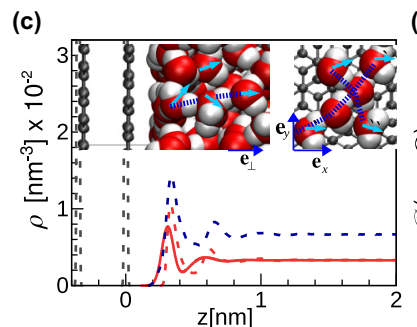

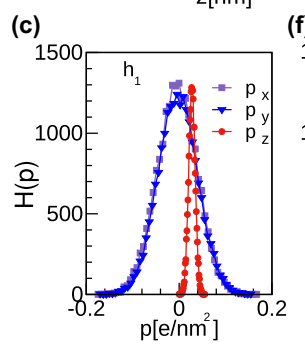

(f) (d)

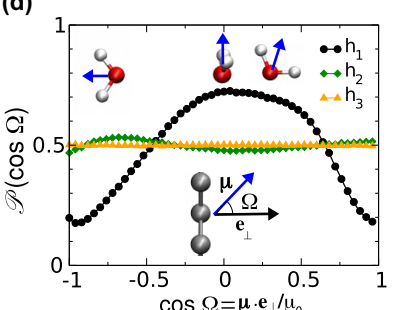

(g)
FIG. 4. The graphene-water interface from experiments and molecular simulations. (a) The measured XR intensities of epitaxial graphene (EG) grown on $\mathrm{SiC}(\mathrm{EG} / \mathrm{SiC})$ in air (blue circles) and $\mathrm{EG} / \mathrm{SiC}$ in deionized water (red circles) are shown with their best fits, $\chi^{2}=1.2$ in air (blue line) and $\chi^{2}=1.6$ in DIW (red line), and the expected intensity from an ideally terminated $\mathrm{SiC}$ substrate for reference (gray line); the Bragg peaks of the $\mathrm{SiC}$ substrate and graphene film are indicated. (b) The best-fit real-space electron density profile (solid lines) reveals a modulated water structure on graphene in agreement with the prediction of molecular dynamics (MD) simulations (dashed lines). (c) The intrinsic water density profiles next to a single uniform graphene surface from the experiment (red solid line) and from MD simulations (dashed lines; $\mathrm{O}$, red; $\mathrm{H}$, blue; $\mathrm{C}$, gray); the insets show MD snapshots of the water-graphene interface along the surface normal direction (left) and in the plane within $z<0.5 \mathrm{~nm}$ (right); the arrows represent the water dipole moment orientation; and the dashed lines represent temporary hydrogen bonds. (d) Water orientation probability distribution function $\mathscr{P}(\cos \Omega)$ within different water regions: $h_{1} \equiv\{0 \leqslant z<0.5 \mathrm{~nm}\}, h_{2} \equiv\{0.5 \mathrm{~nm} \leqslant z<$ $1 \mathrm{~nm}\}$, and $h_{3} \equiv\{1 \mathrm{~nm} \leqslant z<1.5 \mathrm{~nm}\}$ [see Fig. 1(a)]. The water orientation is given by $\cos \Omega$, where $\Omega$ is the angle between a water molecule dipole moment $(\mu)$ and a unitary vector normal to the graphene surface $\left(\mathbf{e}_{\perp}\right) ; \mu_{0} \equiv|\mu|=0.0489 e \mathrm{~nm}$ is the dipole moment of the SPC/E water model, where $e$ is the positive elementary charge; from left to right the three insets show representative configurations of water for $\cos (\Omega)=-1,0$, and $\cos (\Omega) \gtrsim 0$, respectively. [(e)-(g)] The histograms of the instantaneous water polarization components $H\left(\mathrm{p}_{i}\right)(i=x, y, z) . \mathrm{P}_{x}$ and $\mathrm{P}_{y}$ are always equal to zero. The polarization histograms in the $z$ direction are persistently narrower than in the $x$ and $y$ directions up to a water layer thickness of $L_{\mathrm{w}} \approx 120 \mathrm{~nm}$.

water profile with density peaks that correlate with the locations of the graphene layers. The XR best-fit structure 
(a)

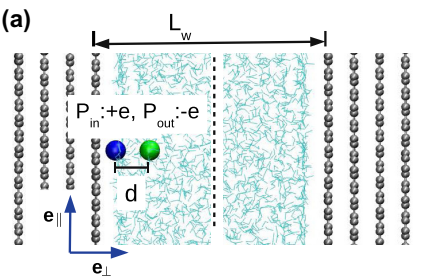

(b)
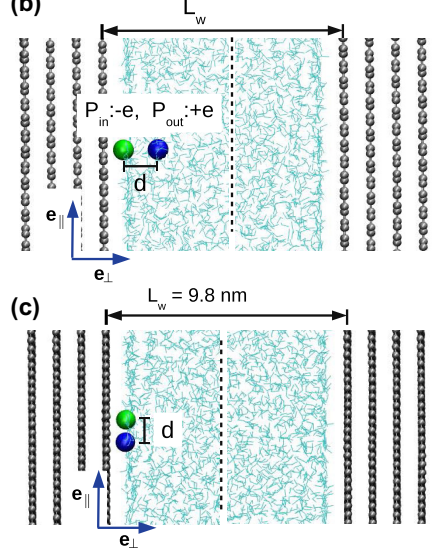

(d)

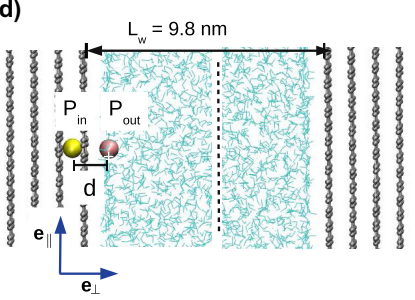

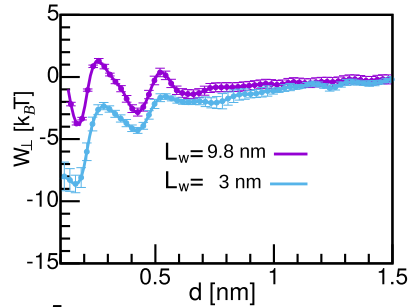
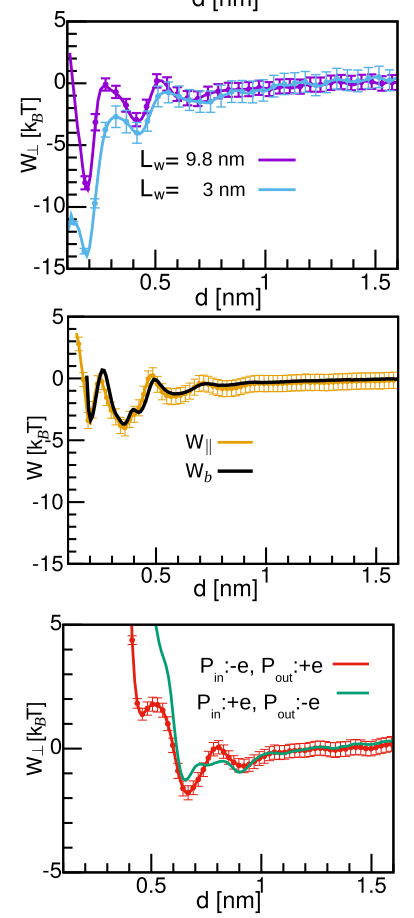

FIG. 5. The ion-ion interaction under confinement is directional, nonequivalent upon permutation of the ions' position, and confinement dependent $\left(L_{\mathrm{w}}\right)$. The potential of mean force profiles along the graphene surface normal direction $\left(W_{\perp}\right)$ are investigated as a function of the ion-ion separation distance $d$. The two ions (designated as $P_{\text {in }}$ and $\left.P_{\text {out }}\right)$ are placed at different heights $z$ above the graphene surface and the two ions have the same $x$ and $y$ coordinates; the $P_{\text {in }}$ ion is fixed at $z_{\text {in }} \approx 0.28 \mathrm{~nm}$. The ion positions are exchanged such that in panel (a) $P_{\text {in }}=+e$ and $P_{\text {out }}=-e$ while in panel (b) $P_{\text {in }}=-e$ and $P_{\text {out }}=$ $+e$. The right-hand side panels show the anion-cation potential of mean force at two confining separation distances, $L_{\mathrm{w}}=3 \mathrm{~nm}$ (light blue line) and $9.8 \mathrm{~nm}$ (purple line). The attractive interactions are nonreciprocal upon exchange the ion's positions and are enhanced by decreasing $L_{\mathrm{w}}$. (c) The in-plane potential of mean force profile $\left(W_{\|}\right)$ between two ions fixed at $z \approx 0.28 \mathrm{~nm}$ and with variable separation distance $d\left(L_{\mathrm{w}}=9.8 \mathrm{~nm}\right.$, yellow line $)$ is similar to that in the bulk $W_{b}$ (black line). (d) The intercalation of one of the ions between the $G_{2}$ and $\mathrm{G}_{3}$ graphene layers leads to predominantly repulsive forces. For the $W_{\perp}$ calculations, $d$ is varied along the surface normal direction, while $d$ is varied along the surface parallel direction for the $W_{\|}$ calculations; in the bulk $W_{b}$ is isotropic.

shows water adsorbed at $\approx 0.31 \pm 0.03 \mathrm{~nm}$ above each exposed graphene surface while MD predicts the adsorbed water height to be $0.33 \mathrm{~nm}$, both of which are consistent with a slightly hydrophobic interface [53,54].

Zhou et al. found, via XR measurements, a significantly reduced water height of $0.23 \mathrm{~nm}$ on the $\mathrm{G}_{0}$ buffer layer compared to a height of $0.32 \mathrm{~nm}$ above free-standing graphene, which suggested that the $\mathrm{G}_{0}$ layer exhibits a hydrophilic character. In contrast, their ab initio molecular dynamics (AIMD) results showed $\mathrm{a} \approx 0.02 \mathrm{~nm}$ decrease in the water height above $\mathrm{G}_{0}(z \approx 0.31 \mathrm{~nm})$ compared to that above free-standing graphene layers $(z=0.33 \mathrm{~nm})$, which is in agreement with our XR results. Zhou et al. attributed the discrepancy in $\mathrm{G}_{0}$ water height between their XR and AIMD results to surface defects in their EG/SiC sample, which likely also explains the observations in the present study. We discuss sources of the $\mathrm{G}_{0}$ water height discrepancy in greater detail in Appendix C. Finally, MD predicts a broadening of the first hydration layer for thicker graphene regions (i.e., on $\mathrm{G}_{3}$ where the total thickness of the graphene slab is $\approx 1 \mathrm{~nm}$ ), a phenomenon not observed in the XR results, with the root mean square (rms) width of water on $\mathrm{G}_{3}$ being most consistent with the experimental results (see Appendix C). Potential explanations of the subtle differences between our MD prediction and XR results may reflect different interactions between water and graphene of various thicknesses and finite-size effects of the simulation compared to the relatively large area of the XR measurement (see Appendix C).

The intrinsic water structure is the density profile corresponding to a uniform graphene surface [see Fig. 1(a)] extracted from XR measurements. Figure 4(c) shows a comparison of the intrinsic water density profiles from XR experiments and MD simulations. Both the XR and MD oxygen density profiles reveal a first hydration layer $(z \approx$ $0.3 \mathrm{~nm}$ ) with a peak density that is more than twice that of the bulk. The density oscillations decay rapidly with a small secondary hydration layer at $z \approx 0.6 \mathrm{~nm}$ and a nearly bulk-like third hydration layer at $z \approx 1 \mathrm{~nm}$ in both $\mathrm{XR}$ and MD profiles. The atomic positions of hydrogen are calculated via MD simulations only due to the weak scattering of $\mathrm{x}$ rays from hydrogen atoms. As such, the agreement between experimental and computational oxygen distributions suggests a layered proton distribution (according to our MD simulations) leading to a nonzero interfacial water polarization. The position of the oxygen layers relative to the graphene surface (validated by the experiment) are used to build the histograms of the interfacial water polarization which is employed to explain the electrostatic interfacial interactions (see below).

The location of the hydrogen density peak at the first hydration layer coincides with the oxygen density peak but is broader, suggesting that a fraction of the water dipole moments are oriented perpendicularly away from the graphene surface. This is partially driven by transient hydrogen bonds between water molecules of the first and second hydration layers [see left inset in Fig. 4(c)] and the inability of water molecules to form hydrogen bonds with the graphene surface. The water orientation is quantified by the distribution function of its dipole orientation $\cos \Omega$ with respect to the graphene surface normal $\mathbf{e}_{\perp}$ [Fig. 4(d)]. The distribution reveals a first hydration layer in the region $h_{1}(z<0.5 \mathrm{~nm})$ with the majority of water dipole moments oriented parallel to the graphene surface ( $\cos \Omega=0$ ). However, the asymmetry in the distribution exhibits a slight preference of the water dipole moment to orient away from the graphene surface. The water molecules in the $h_{1}$ region form a hydrogen bond network which is a characteristic behavior of water near hydrophobic 
molecules [55] [right inset in Fig. 4(c)]. In the region defined at $0.5 \mathrm{~nm}<z \leqslant 1 \mathrm{~nm}\left(h_{2}\right)$, the preferential orientation of water molecules is diminished [Fig. 4(c)], and from $z>1 \mathrm{~nm}$ all the water dipole moment orientations are equally probable $(1 \mathrm{~nm}<z \leqslant 1.5 \mathrm{~nm})$.

We now examine in further detail the interfacial water polarization. Recent experiments investigated the out-of-plane permittivity $\left(\varepsilon_{\perp}\right)$ of water confined between two flat surfaces of graphite and hexagonal boron nitride. The experiments reveal that $\varepsilon_{\perp} \approx 2$ within an interfacial layer of two or three molecules thick (referred to as the electrically dead layer) which is considered to have a "vanishingly small polarization" [12]. Here, in contrast, we observe a nonzero persistent water polarization in the $z$ direction within the interfacial region. Figures $4(\mathrm{e})-4(\mathrm{~g})$ show the histograms of the instantaneous polarization $H(\mathrm{p})$ within the regions $h_{1}, h_{2}$, and $h_{3}$, respectively, defined to capture the different hydration layers. The average polarization in the $z$ direction is $P_{z} \equiv\left\langle\mathrm{p}_{z}\right\rangle \approx$ $0.03 e / \mathrm{nm}^{2}$ in the $h_{1}$ region [Fig. 4(e)], $P_{z} \approx-0.004 e / \mathrm{nm}^{2}$ in the $h_{2}$ region [Fig. 4(f)], and $P_{z}=0$ in the $h_{3}$ region [Fig. 4(g)] and for $z \gtrsim 1 \mathrm{~nm}$, while $P_{x}$ and $P_{y}$ are always equal to zero. In particular, we highlight the nonvanishing polarization $P_{z}$ within the $h_{1}$ region, which is fundamental to understanding the electrostatic interactions near an interface (see the next section).

The dipole fluctuations are known to be suppressed along the surface normal direction [56]. Here, the suppression is seen as a much narrower histogram for the polarization in the $z$ direction than in the $x$ and $y$ directions. The polarization suppression implies that every fluctuation of $\mu_{z}$ is nearly canceled by an antiparallel component. The suppression is observed across the entire water region and up to a water layer thickness of $L_{\mathrm{w}} \approx 120 \mathrm{~nm}$. Additionally, we investigated the liquid-vapor interface by removing the graphene surfaces, and we found that the dipole moment fluctuations in the $z$ direction remain suppressed. This observation shows that the suppression is not only observed at a liquid-solid interface, and it is not related to the chemical structure of the confining surfaces.

\section{Ion-ion interactions in confinement}

We look into a direct force analysis between an anion and a cation in bulk water, near the graphene surface, and between an ion in the water and an ion intercalated between the graphene layers. Ion intercalation is the common mechanism for Faradaic energy storage (e.g., lithium ion batteries with graphitic anodes) [57], while ion adsorption occurs in capacitive energy storage systems. Figures 5(a)-5(c) show that confinement enhances the ion-ion interaction along the surface normal direction, which is more pronounced at $L_{\mathrm{w}}=$ $9.8 \mathrm{~nm}$ than in the bulk and even more pronounced by decreasing the water layer thickness to $L_{\mathrm{w}}=3 \mathrm{~nm}$; the minimum of the PMF decreases by about $5 k_{B} T$ by decreasing $L_{\mathrm{w}}$ from 9.8 to $3 \mathrm{~nm}$. The enhancement of the ion-ion interaction near the graphene surface is consistent with observations near a hydrophobic surface [10]. Furthermore, the effective ion-ion interaction under confinement is nonreciprocal, i.e., the potential of mean force is not equivalent upon exchange of the ions' positions [see Figs. 5(a) and 5(b)], where it is seen that the minimum of the PMF decreases by about $5 k_{B} T$ by exchanging the ions' positions. This nonreciprocal behavior of the anion-cation interactions is also observed when polarizable models of $\mathrm{Na}^{+} \mathrm{Cl}^{-}$ions, polarizable water, and polarizable graphene are used in the simulations (see Appendix A). The ion-ion interaction in the surface plane direction $W_{\|}$, however, is not significantly different from the ion-ion interaction in bulk [Fig. 5(c)] likely because the ions are less confined in the $x y$ plane. Interestingly, the interaction along the surface normal direction changes from predominantly attractive when both ions are in the aqueous phase [Figs. 5(a) and 5(b)] to completely repulsive when one ion is intercalated within the graphene interlayer space [Fig. 5(d)]. In an isotropic medium, the ion-ion effective force depends only on the ions' separation distance, and the forces are
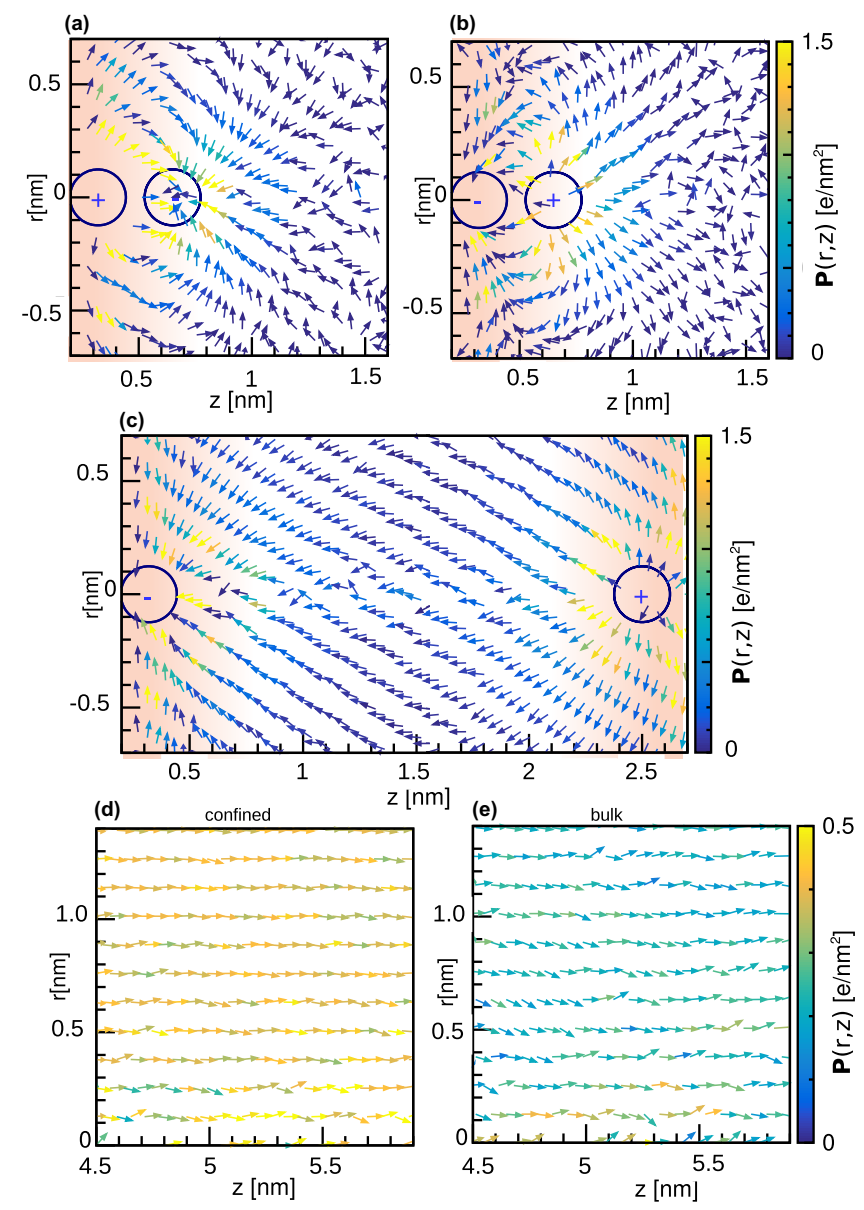

FIG. 6. Water polarization field in confinement affects the ionion effective interaction. The anion-cation separation vector is oriented along the graphene surface normal; (a) the cation is next to the graphene surface at $z_{\text {in }} \approx 0.28 \mathrm{~nm}$ and the anion is at $z_{\text {out }} \approx$ $0.62 \mathrm{~nm}$; (b) the anion is at $z_{\text {in }} \approx 0.28 \mathrm{~nm}$ while the cation is at $z_{\text {out }} \approx 0.62 \mathrm{~nm}$. (c) The anion and cation are placed at a separation distance of $2.2 \mathrm{~nm}$ at the opposite sides of the water layer near the graphene surfaces separated by a water layer of thickness $L_{\mathrm{w}}=3 \mathrm{~nm}$. The water polarization at the middle region between the ions at a separation distance of $8.9 \mathrm{~nm}$ in $(\mathrm{d})$ confinement $\left(L_{\mathrm{w}}=9.8 \mathrm{~nm}\right)$ and in (e) bulk. The bulk system is simulated using periodic boundary conditions in the $x, y$, and $z$ directions in a box of length $L_{z}=20 \mathrm{~nm}$ in the $z$ direction. 
(a)

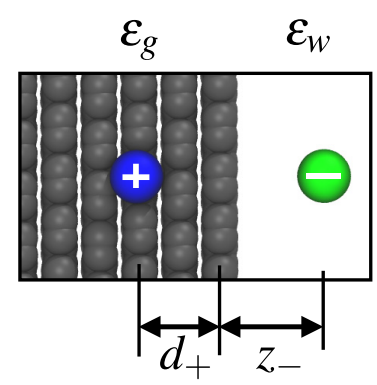

(b)

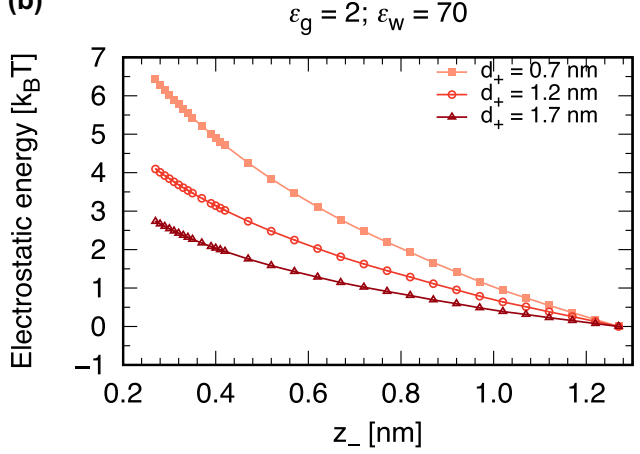

(c)

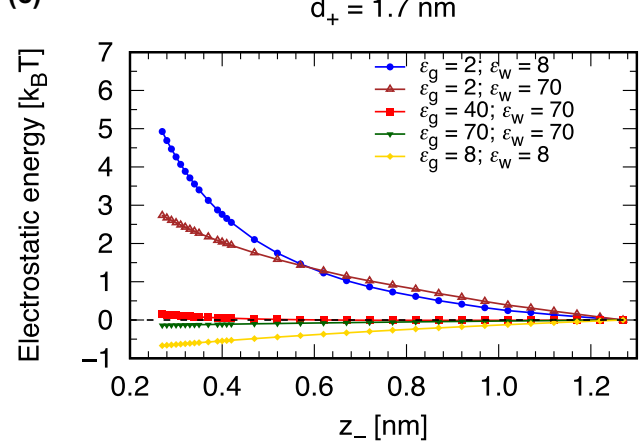

FIG. 7. Coulombic energy as a function of the distance between the two ions computed from the image charge method for the case where the cation is inside the graphene surface. In panel (a), graphene atoms are visualized to guide the eye but not involved in the image charge calculations.

equivalent upon exchange of the ions' positions. However, Figs. 5(a)-5(d) reveal that in confinement the effective ion-ion interaction is directional and position dependent with respect to the graphene surface. The force nonreciprocity cannot be explained by current models of anisotropic dielectric permittivity $\left(\varepsilon_{\|}\right.$and $\left.\varepsilon_{\perp}\right)$, which predict the same dielectric profile regardless of the ion valence and, hence, a symmetric force. We will discuss this point in detail in Subsec. III E.

The nonreciprocal behavior of the ion-ion interfacial interactions and the ion-sign specificity are due to the unsymmetrical response of the interfacial water polarization. The classical theory assumes surface polarization is the same for positive and negative charges whereas our results show the polarization field strongly depends on the sign of the charge. The potential of mean force $W_{c}$ from the MD simulations takes into account the ion-ion direct interaction and the interaction through the surrounding water molecules, which includes the work to reorient the interfacial polarization. The interfacial polarization when the cation is placed on the graphene surface and when the anion is on the surface (exchanged configuration) are shown in Figs. 6(a) and 6(b), respectively. These polarization fields give rise to different ion-ion interactions. The polarization around two ions close to each other is canceled at a short distance $(\lesssim 1.5 \mathrm{~nm})$, which is consistent with the observation around the polar groups of proteins [58]. When the two charges are separated at longer distances, however, the polarization propagates over the whole separation distance [Fig. 6(c)]. The polarization in confinement is enhanced even at larger confinement distances. Figures $6(d)$ and 6(e) show the difference in the water polarization at the middle distance between the two ions separated by about $9.5 \mathrm{~nm}$ in confinement and in bulk, respectively. The magnitude of polarization in confinement is approximately twice and shows less deviation from the perpendicular orientation than the polarization in bulk.

\section{Results from continuum electrostatics theories}

We calculate the ion-ion Coulombic interaction energy as a function of the distance between the two ions arranged in the three configurations in Figs. 5(a)-5(c) using the image charge method (see Subsec. IIC). We assume that the water is a continuum background with the permittivity of $\varepsilon_{1}=\varepsilon_{\mathrm{w}}=70$ and the graphene is a continuum medium of permittivity of $\varepsilon_{2}=\varepsilon_{\mathrm{g}}=2$. The image charge method cannot predict the oscillations found in the potential of mean force in Fig. 5 when surface polarization effects are included. Instead, a purely repulsive interaction is observed assuming a dielectric mismatch at the interface of $\alpha=0.97$. Figure 7 shows the ion-ion interaction energy (from the image charge method) when the cation is placed inside the graphene surface for different combinations of the dielectric constants of the two media forming the interface. It is interesting to see that the continuum theory captures the change from attractive to repulsive for sufficiently large dielectric mismatches. The repulsive behavior is in agreement with the results from umbrella sampling simulations when one ion is intercalated between the graphene layers [see Fig. 5(d)]; however, the interaction energy obtained from the image charge method is the same when the ions' positions are exchanged.

\section{E. Results from the molecular theory of permittivity}

We investigated the interfacial dielectric permittivity employing the molecular theory described in Subsec. II D. The expressions of the permittivity in the direction parallel to the graphene surface $\left[\varepsilon_{\|}(z)\right]$ and the inverse permittivity in the perpendicular direction $\left[\varepsilon_{\perp}^{-1}(z)\right]$ are given by Eqs. (7) and (8), respectively. The ultimate goal of quantifying the interfacial permittivity is to determine the electrostatic interaction between charged species near the interface. We estimate the interfacial ion-ion interaction by means of the Coulomb's potential employing a position-dependent permittivity $\varepsilon(\mathbf{r})$ given by Eq. (8). We see that the interaction potential $W^{\mathrm{C}}$ significantly overestimates the interaction with respect to the potential of mean force from umbrella sampling simulations $W_{\perp}$ (see Fig. 8). As we mentioned above, the potential of mean force from umbrella sampling simulations $W_{\perp}$ is different by exchanging the positions of the two particles (nonreciprocal), whereas here we see that the ion-ion interaction calculated from the permittivity profile from Eq. (8) is the same independently of the ions arrangement (see Fig. 8). This shows that the interaction between two charged particles cannot be described solely in terms of a permittivity function. 

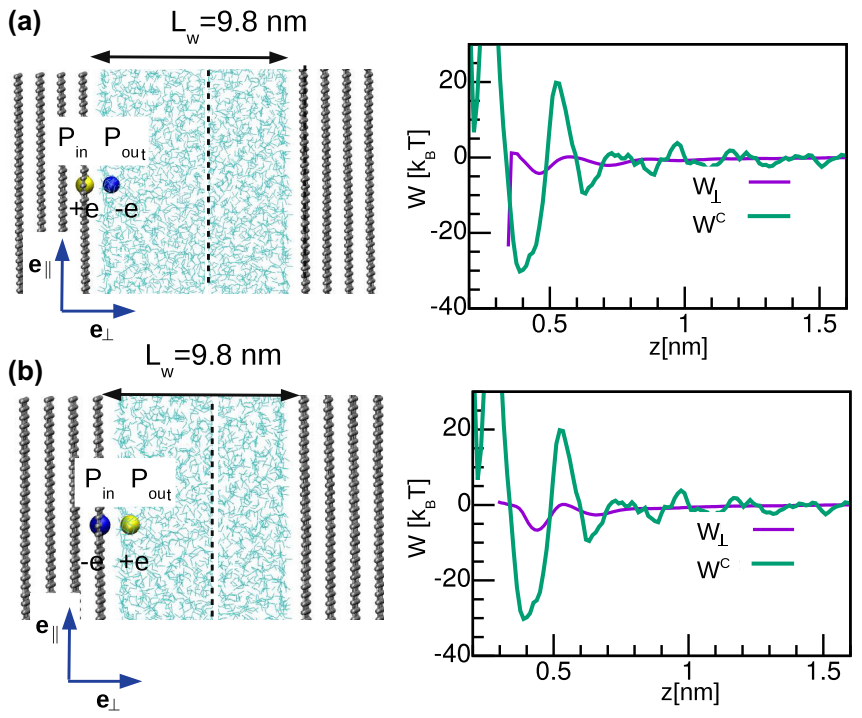

FIG. 8. Interaction between two ions at the water graphene interface along the surface perpendicular direction. The ion at the inner position $P_{\text {in }}$ is placed as part of the outermost graphene layer while the ion at the outer position $P_{\text {out }}$ is into the water phase; in panel (a) $P_{\text {in }}=+e$ and $P_{\text {out }}=-e$ while in panel (b) $P_{\text {in }}=-e$ and $P_{\text {out }}=$ $+e$. The right-hand side panels show the anion-cation potential of mean force where the purple line $\left(W_{\perp}\right)$ represents the potential of mean force calculated via umbrella sampling and the green line is the the interaction potential calculated as $W^{\mathrm{C}}=l_{0} \varepsilon_{\perp}^{-1}(z) / z$, where $l_{0}=e^{2} /\left(4 \pi \varepsilon_{0} k_{B} T\right)$ and the inverse perpendicular permittivity $\varepsilon_{\perp}^{-1}(z)$ is calculated via Eq. (7).

\section{CONCLUSIONS}

Our results show that in confinement the ion-ion effective interaction is directional and position dependent with respect to the graphene surface due to a nonzero persistent interfacial water polarization. We identify this property as nonreciprocity, which describes the directionally dependent interactions and nonequivalent change of interactions upon exchange of the ions' positions near a confining surface. The nonreciprocity implies that ion-ion interactions at the interface do not obey the isotropic and translational symmetries of Coulomb's law and are observed in both polarizable and nonpolarizable models. This phenomenon contrasts with the ion-ion interactions in an isotropic medium (bulk) where the force depends only on the ions' size and separation distance, is not directional, and is equivalent by exchange of the ions' positions. Traditionally, ion specificity is attributed to the internal ion polarization including polarizability associated with ion size. Here, we find that the water polarization plays a central role in the behavior of ions near interfaces. Namely, the water polarization around ions and near the interface alters electrostatic interactions, leading to nonequivalent ion-interface interactions upon exchange of the ion charge sign even if the ions have equal size. This nonsymmetrical water polarization affects the understanding of ion-differentiation mechanisms such as ion selectivity and ion specificity. The agreement between XR experimental measurements and MD simulations of polarizable and nonpolarizable models suggests a layered hydrogen structure which leads to the interfacial wa- ter polarization. We find that the water structure near the graphene-water interface, however, is not enough to infer electrostatic interactions near the interface. The current models based on the anisotropic dielectric permittivity in confinement (obtained via the water structure) cannot explain the nonreciprocal ion-ion interactions found here. Our simulations reveal that the valence (charge) of the ions, irrespectively of other ion specific effects, is responsible for the nonreciprocal interactions. Molecular interactions near interfaces and in confinement are related to a variety of processes including chemical reactions, adsorption, and biological molecular recognition. The insights gained here need to be considered in the understanding of processes based on asymmetric ionic adsorption and interactions at heterogeneous interfaces such as proteins.

\section{ACKNOWLEDGMENTS}

F.J.-A., T.D.N., and M.O.d.1.C. were supported by the Department of Energy (DOE), Office of Basic Energy Sciences under Contract No. DE-FG02-08ER46539. The computational work was done with the support of the Sherman Fairchild Foundation. This work was supported, in part, by the Midwest Integrated Center for Computational Materials (MICCoM) as part of the Computational Materials Sciences Program funded by the U.S. Department of Energy, Office of Science, Basic Energy Sciences, Materials Sciences and Engineering Division (Grant No. 5J-30161-0010A). K.J.H. gratefully acknowledges support from the U.S. Department of Defense through the National Defense Science and Engineering Graduate Fellowship (NDSEG) Program and from the Ryan Fellowship at Northwestern University International Institute of Nanotechnology. We thank Dr. Jon Emery (Northwestern University) and Dr. D. Kurt Gaskill (Naval Research Laboratory) for the graphene sample and for discussions on the structure of graphene. X-ray reflectivity measurements were performed at beamline 33-ID-D of the Advanced Photon Source at Argonne National Laboratory (ANL), a U.S. DOE Office of Science User Facility operated by ANL under Contract No. DE-AC02-06CH11357.

\section{APPENDIX A: POLARIZABLE MODELS}

The induced polarization of atoms results from the deformation of the electronic cloud due to a local electric field and it is known to be relevant in some interfacial phenomena [59]. At first approximation, the main contribution of the induced polarization is from the induced dipole moment $\left(\mu_{\text {ind }}\right)$ of atoms, ions, and molecules due to the local electric field $\mathbf{E}^{\prime}(\mathbf{r})$, given by

$$
\boldsymbol{\mu}_{\text {ind }}=\alpha_{i} \mathbf{E}^{\prime}(\mathbf{r})
$$

where $\alpha_{i}$ is the polarizability, which is a distinctive property for each atom and ion. To account for polarization effects, we employ the SWM4-NDP polarizable water model [60] and the corresponding force field parameters for the polarizable $\mathrm{Na}^{+}$and $\mathrm{Cl}^{-}$ions [61]. Polarizable graphene is modeled by including the polarizability derived from DFT calculations [62] in our graphene model. The water molecule has a fixed $\mathrm{HOH}$ geometry, bearing two positive charges at the hydrogen 
centers and the negative charge is placed at a fixed distance from the oxygen center, shifted along the molecule's axis of symmetry. The induced polarization is based on the Drude particle model of charge $q_{D}$, attached to the center of the polarizable atoms (or ions) through a harmonic potential. The Drude particle's charge is balanced by the positive charge of the core such that, $q_{C}+q_{D}=0$ for neutral atoms and $q_{C}+q_{D}=q_{\text {ion }}$ for ions, where $q_{\text {ion }}$ is the ionic charge. The spring constant $k_{D}$, the polarizability $\alpha_{i}$, and the charge of the Drude particle $q_{D}$ are related by

$$
\alpha_{i}=q_{D}^{2} / k_{D} \text {. }
$$

The polarizabilities employed in our calculations are $\alpha_{\mathrm{g}}=$ $1.139 \AA^{3}$ for graphene carbon atoms, $\alpha_{\mathrm{w}}=0.978 \AA^{3}$ for water oxygen atoms, $\alpha_{\mathrm{Na}^{+}}=0.157 \AA^{3}$ for $\mathrm{Na}^{+}$, and $\alpha_{\mathrm{Cl}^{-}}=$ $3.969 \AA^{3} \mathrm{Cl}^{-}$. Integration of the equations of motion is performed by means of the extended Lagrangian algorithm [63], which consists in assigning a small mass to the Drude particles $m_{D}$ taken from the atomic masses, in such a way that the mass of the core is $m_{i}-m_{D}$. The Drude particles are simulated at a much smaller temperature than the whole system to fulfill the Born-Oppenheimer minimum energy condition. The Drude particles mass is $m_{D}=0.4 \mathrm{~g} / \mathrm{mol}$. A dual Nosé-Hoover thermostat is used to maintain the Drude particles temperature at $T_{D}=1 \mathrm{~K}$ and the system at $T=298 \mathrm{~K}$. We simulated a set of systems similar to that described in Subsec. II A, namely, a water layer of thickness $L_{\mathrm{w}} \approx 9.8 \mathrm{~nm}$, formed by $N_{\mathrm{w}}=8060$ water molecules, and confined between two graphene surfaces formed by four graphene layers each [see Fig. 1(a)]. A time step of $1 \mathrm{fs}$ is employed for integration of the equations of motion of the systems without ions. When ions are present, the time step is $0.2 \mathrm{fs}$. The simulation protocols are similar to those described in Sec. II. Consideration of polarizability of the water molecules implies an additional dipole moment contribution to the permanent dipole moment.

First, we examine the water structure near the graphene surface considering four different combinations, namely, (a) polarizable water and polarizable graphene, (b) polarizable water and nonpolarizable graphene, (c) nonpolarizable water and polarizable graphene, and (d) nonpolarizable water and nonpolarizable graphene. The density profiles as a function of the perpendicular distance to the graphene surface are shown in Fig. 9. The four combinations predict qualitatively similar profiles exhibiting peaks at the same location, approximately. Our results are consistent with previous studies which show that the graphene polarizability does not significantly affect the water density profile $[62,64]$. Here, the polarizable water model predicts higher peaks the nonpolarizable water. Analysis of the polarization field shows a persistent interfacial polarization qualitatively similar in the four combinations (see Fig. 10).

In Fig. 11, we analyze the effective interaction between two ions $\left(\mathrm{Na}^{+}\right.$and $\left.\mathrm{Cl}^{-}\right)$in water nearby the graphene surface. All the components in the system (water, ions, and graphene) are polarizable. The ions are placed at different heights above the graphene surface aligned perpendicularly to the graphene surface in a similar way as in Figs. 5(a) and 5(b). The potential of mean force (PMF) is calculated in two cases: (1) the $\mathrm{Na}^{+}$ ion is placed on the graphene surface while the $\mathrm{Cl}^{-}$ion is at different heights from the graphene surface and (2) the ions'

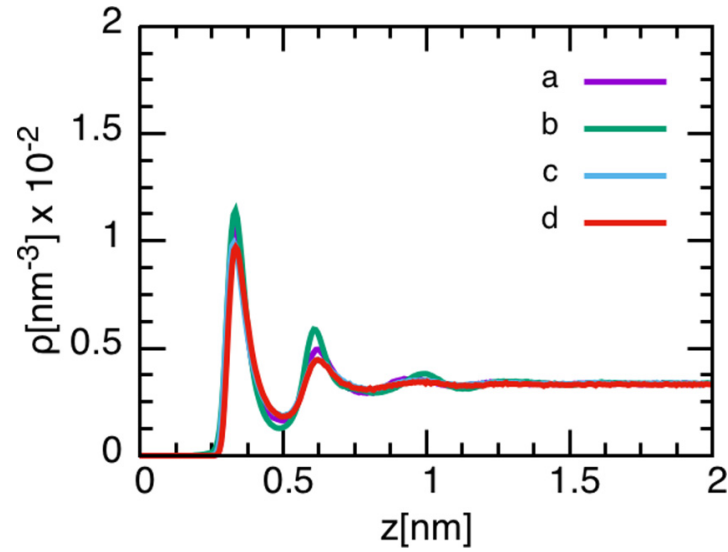

FIG. 9. Effect of water and graphene polarizabilities on the water density profile. (a) Polarizable water $\left(\alpha_{\mathrm{w}}=0.978 \AA^{3}\right)$ and polarizable graphene $\left(\alpha_{\mathrm{g}}=1.139 \AA^{3}\right)$, (b) polarizable water $\left(\alpha_{\mathrm{w}}=\right.$ $\left.0.978 \AA^{3}\right)$ and nonpolarizable graphene $\left(\alpha_{\mathrm{g}}=0\right)$, (c) nonpolarizable water $\left(\alpha_{\mathrm{w}}=0\right)$ and polarizable graphene $\left(\alpha_{\mathrm{g}}=1.139 \AA^{3}\right)$, and (d) nonpolarizable water $\left(\alpha_{\mathrm{w}}=0\right)$ and nonpolarizable graphene $\left(\alpha_{\mathrm{g}}=0\right)$. The water layer thickness is $L_{\mathrm{w}}=9.8 \mathrm{~nm}$.

positions are exchanged. The PMF profiles are qualitatively different from those for nonpolarizable ions [see Figs. 5(a) and $5(\mathrm{~b})$ for $L_{\mathrm{w}}=9.8 \mathrm{~nm}$ ]. For example, the profiles exhibit less pronounced oscillations when $\mathrm{Na}^{+}$is on the graphene surface (purple line in Fig. 11) than for the nonpolarizable ions discussed Figs. 5(a) and 5(b). The difference in energy when the ion positions are exchanged, at the minimum of the PMF, is $5 k_{B} T$ in the fully polarizable system (see Fig. 11). This is similar in both magnitude and sign to that observed for the nonpolarizable system [see Figs. 5(a) and 5(b) for $\left.L_{\mathrm{w}}=9.8 \mathrm{~nm}\right]$. Hence, the nonreciprocal behavior of the ion-
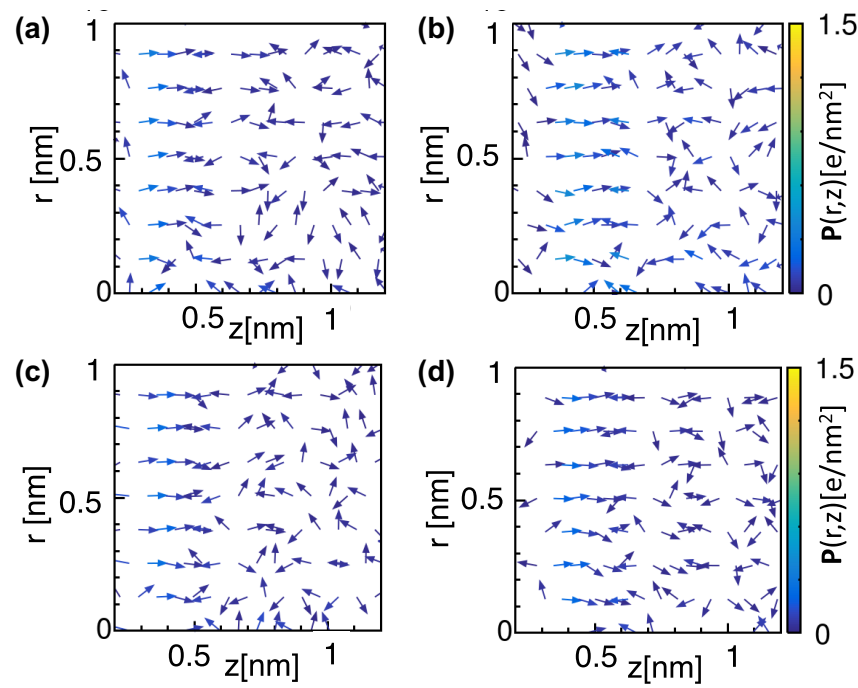

FIG. 10. Polarization field in polarizable models. (a) Polarizable water $\left(\alpha_{\mathrm{w}}=0.978 \AA^{3}\right)$ and polarizable graphene $\left(\alpha_{\mathrm{g}}=\right.$ $\left.1.139 \AA^{3}\right)$, (b) polarizable water $\left(\alpha_{\mathrm{w}}=0.978 \AA^{3}\right)$ and nonpolarizable graphene $\left(\alpha_{\mathrm{g}}=0\right)$, (c) nonpolarizable water $\left(\alpha_{\mathrm{w}}=0\right)$ and polarizable graphene $\left(\alpha_{\mathrm{g}}=1.139 \AA^{3}\right)$, and (d) nonpolarizable water $\left(\alpha_{\mathrm{w}}=0\right)$ and nonpolarizable graphene $\left(\alpha_{\mathrm{g}}=0\right)$. 


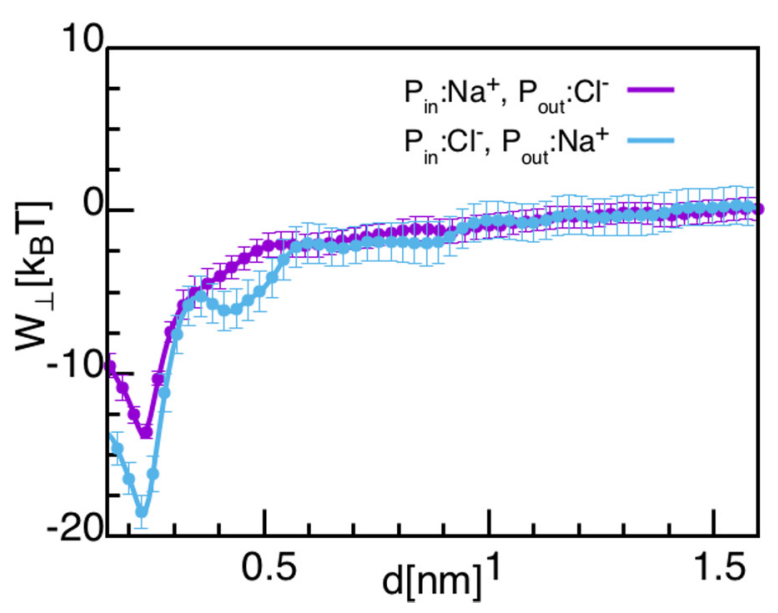

FIG. 11. Interaction between polarizable $\mathrm{Na}+$ and $\mathrm{Cl}^{-}$ions at the water-graphene interface. The potential of mean force $\left(W_{\perp}\right)$ as a function of the ion-ion separation distance $d$ along the graphene surface normal direction. The two ions $\left(P_{\text {in }}\right.$ and $\left.P_{\text {out }}\right)$ are placed at different heights $z$ above the graphene surface at the same $x$ and $y$ coordinates [see Figs. 5(a) and 5(b)]. The ion positions are exchanged such that in panel (a) $P_{\text {in }}=\mathrm{Na}+$ and $P_{\text {out }}=\mathrm{Cl}^{-}$while in panel (b) $P_{\text {in }}=\mathrm{Cl}^{-}$and $P_{\text {out }}=\mathrm{Na}^{+}$. The ions' polarizabilities are $\alpha_{\mathrm{Na}^{+}}=$ $0.157 \AA^{3}$ and $\alpha_{\mathrm{Cl}^{-}}=3.969 \AA^{3}$ for $\mathrm{Na}^{+}$and $\mathrm{Cl}^{-}$, respectively. The water polarizability is $\alpha_{\mathrm{w}}=0.978 \AA^{3}$ and the graphene polarizability is $\alpha_{\mathrm{g}}=1.139 \AA^{3}$. The water layer is confined between two graphene surfaces separated by $L_{\mathrm{w}}=9.8 \mathrm{~nm}$.

ion interactions is present in both systems with polarizable and nonpolarizable atoms and ions.

\section{APPENDIX B: LINEAR RESPONSE THEORY FOR THE DIELECTRIC RESPONSE}

An electric field $\mathbf{E}$ applied in a dielectric material induces a polarization due to a separation of the bound charges in the material (atomic nuclei and their electrons). The macroscopic field $\mathbf{D}$ is called the electric displacement and is given as

$$
\mathbf{D}=\varepsilon_{0} \mathbf{E}+\mathbf{P},
$$

where $\mathbf{P}$ is the polarization density. Linear response theory assumes that for a weak applied electric field, the induced electric polarization is proportional to the magnitude of the applied field

$$
\mathbf{P}=\varepsilon_{0} \underline{\chi} \cdot \mathbf{E},
$$

where $\chi$ is the susceptibility tensor, which is related to the dielectric permittivity tensor $\underline{\varepsilon}(\mathbf{r})$ by

$$
\underline{\varepsilon}=\mathbf{I}+\underline{\chi} .
$$

$\mathbf{I}$ is the identity matrix. $\mathbf{D}$ is then expressed as

$$
\mathbf{D}=\varepsilon_{0} \underline{\varepsilon} \cdot \mathbf{E} .
$$

Using index notation, Eq. (B4) in Cartesian coordinates can be expressed as

$$
D_{\alpha}=\varepsilon_{0} \sum_{\beta=x, y, z} \varepsilon_{\alpha \beta} E_{\beta}
$$

In simple materials, the dielectric behavior is isotropic, leading to a diagonal dielectric tensor with three equal components. Under nanoscale confinement, the dielectric response is anisotropic, and the components of the dielectric tensor are not equal. In the next part, we present the main steps to derive the expression for the dielectric permittivity for a nonuniform medium in one dimension.

Our treatment follows closely the work by Feller and Stern [14], Ballenegger and Hansen [13], and Netz' group [15]. The basic idea is to compute the response of a dielectric medium to a static, external, and uniform electric field $\mathbf{E}$, combining descriptions from statistical mechanics and continuum electrostatics. By combining both approaches, we are able to derive the expression of the local permittivity.

Let $\Delta \mathbf{E}(\mathbf{r})$ be the change in the mean local electric field inside the dielectric. When the external electric field $\mathbf{E}$ is turned on, the change is due to both the external field itself and the dipoles within the medium. $\mathbf{E}_{0}(\mathbf{r})$ and $\mathbf{P}_{0}(\mathbf{r})$ are, respectively, the mean electric field and the mean local polarization with no applied external field $\left[\mathbf{E}_{0}(\mathbf{r})\right.$ is zero if $\left.\mathbf{P}_{0}(\mathbf{r})\right]$. In the linear response regime, the change in the local polarization $\Delta \mathbf{P}(\mathbf{r})$ and the change in the total electric field $\Delta \mathbf{E}(\mathbf{r})$ are related by

$$
\Delta \mathbf{P}(\mathbf{r})=\underline{\chi}(\mathbf{r}) \cdot \Delta \mathbf{E}(\mathbf{r}),
$$

where $\chi(\mathbf{r})$ is the local susceptibility tensor which is related to the local dielectric permittivity tensor $\underline{\varepsilon}(\mathbf{r})$ as

$$
\underline{\varepsilon}(\mathbf{r})=\underline{\chi}(\mathbf{r})+\mathbf{I} .
$$

The expression for $\Delta \mathbf{P}(\mathbf{r})$ is derived from statistical mechanics while the expression for $\Delta \mathbf{E}(\mathbf{r})$ is from macroscopic electrostatics.

\section{Microscopic description}

We consider a classical system in a microstate $\Gamma$ described by the Hamiltonian $H(\Gamma)$. In general, the dipole moment $\mathbf{m}$ changes from point to point within the dielectric. Hence, the instantaneous polarization density at $\mathbf{r}, \mathbf{p}(\mathbf{r})$ (also known as electric polarization, or simply polarization) is given by

$$
\mathbf{p}(\mathbf{r})=\frac{\Delta \mathbf{m}}{\Delta V} .
$$

The system's total dipole $\mathbf{M}$ is given by

$$
\mathbf{M}=\int \mathbf{p}(\mathbf{r}) d V .
$$

The mean polarization is expressed as

$$
\mathbf{P}(\mathbf{r})=\langle\mathbf{p}(\mathbf{r})\rangle=\frac{\int \mathbf{p}(\mathbf{r}) e^{-\beta H} d \Gamma}{\int e^{-\beta H} d \Gamma} .
$$

Let $H$ and $H^{\prime}$ be, respectively, the system's Hamiltonian when there is no applied external electric field and when the electric field is turned on. In linear response theory, $H^{\prime}$ can be expressed as $H^{\prime}=H-\mathbf{M} \cdot \mathbf{E}$, and the change in the mean polarization as

$$
\begin{aligned}
\Delta \mathbf{P}(\mathbf{r}) & =\mathbf{P}_{E}(\mathbf{r})-\mathbf{P}(\mathbf{r}) \\
& =\langle\mathbf{p}(\mathbf{r})\rangle_{E}-\langle\mathbf{p}(\mathbf{r})\rangle \\
& =\frac{\int(\mathbf{p}(\mathbf{r})-\langle\mathbf{p}(\mathbf{r})\rangle) e^{-\beta H^{\prime}} d \Gamma}{\int e^{-\beta H^{\prime}} d \Gamma} .
\end{aligned}
$$


In the weak electric field regime, we linearize the above expression to get

$$
\Delta P_{\alpha}(\mathbf{r})=\beta \sum_{\gamma=x, y, z}\left[\left\langle p_{\alpha}(\mathbf{r}) M_{\gamma}\right\rangle-\left\langle p_{\alpha}(\mathbf{r})\right\rangle\left\langle M_{\gamma}\right\rangle\right] E_{\gamma},
$$

where we have switched to express the components $\alpha=x, y, z$ of the vector $\Delta \mathbf{P}(\mathbf{r})$, and the statistical average is performed in the zero electric field regime. Equation (B12) involves the correlation between a fluctuation in the local polarization density $\mathbf{m}(\mathbf{r})$ and a fluctuation in the global dipole moment $\mathbf{M}$ of the system.

To calculate the permittivity tensor via Eq. (B6), it is necessary to derive an expression for $\Delta \mathbf{E}$. The calculation of $\Delta \mathbf{E}$ is performed by considering the dipolar contributions from every molecule and from each image cell in a system where periodic boundary conditions are assumed in the $x, y$, and $z$ directions. By doing so, Feller and Stern arrived at the following expressions [14] for the dielectric permittivity profile in the parallel $\left[\varepsilon_{\|}(z)\right]$ and perpendicular $\left[\varepsilon_{\perp}(z)\right]$ directions to the graphene surface given by Eqs. (7) and (8), respectively.

\section{APPENDIX C: EXPERIMENTAL DETAILS}

\section{X-ray reflectivity background and model}

The specular XR signal $R(Q)$ is directly related to the laterally averaged real-space electron density profile $\rho(z)$ via Fourier transform as

$$
R(Q)=T(Q) B(Q)\left(\frac{4 \pi r_{e}}{A_{U C} Q}\right)^{2}\left|\int_{-\infty}^{\infty} \rho(z) e^{i Q z} d z\right|^{2},
$$

where $T(Q)$ is the angle-dependent transmission of $\mathrm{x}$ rays through the sample cell, $B(Q)$ accounts for the effects of surface roughness [65], $r_{e}=2.82 \times 10^{-5} \AA$ is the classical electron radius, and $A_{U C}$ is the unit cell area of the substrate ( $\mathrm{SiC}$ in the present case); $Q$ is the vertical momentum transfer as in Fig. 2(a). Because of the loss of phase information inherent in the XR measurement, it is not possible to determine $\rho(z)$ by inverse FT. Instead, it is determined by optimizing a model wherein the $j$ th atomic layer along the $z$ direction is represented by a Gaussian with fitting parameters of position, width, and coverage as described in Eq. (10). The parameter values and their uncertainties are determined via nonlinear least squares fitting following the Levenberg-Marquardt algorithm until the level of agreement [Eq. (12)] between the calculated model-dependent reflectivity, $R(Q)_{\text {calc }}$, and the experimental data, $R(Q)_{\exp }$, converges.

\section{2. $\mathrm{DIW} / \mathrm{EG} / \mathrm{SiC}$ interface structure}

Figure 12 shows an AFM height scan of the EG/SiC sample in air. It reveals a smooth sample surface (average surface roughness $=38 \mathrm{pm}$ ) with lateral terrace widths of $\approx 1 \mu \mathrm{m}$. We also see several layers of graphene on each terrace with a mean thickness of about $6 \AA$ and a maximum thickness of about $12 \AA$ (Fig. 12, top). Because of the limited resolution of the AFM, its chemical insensitivity, and the fact that it does not see the layers below the surface, we cannot determine the number of graphene layers precisely from the AFM. Nevertheless, this qualitative picture provides an additional
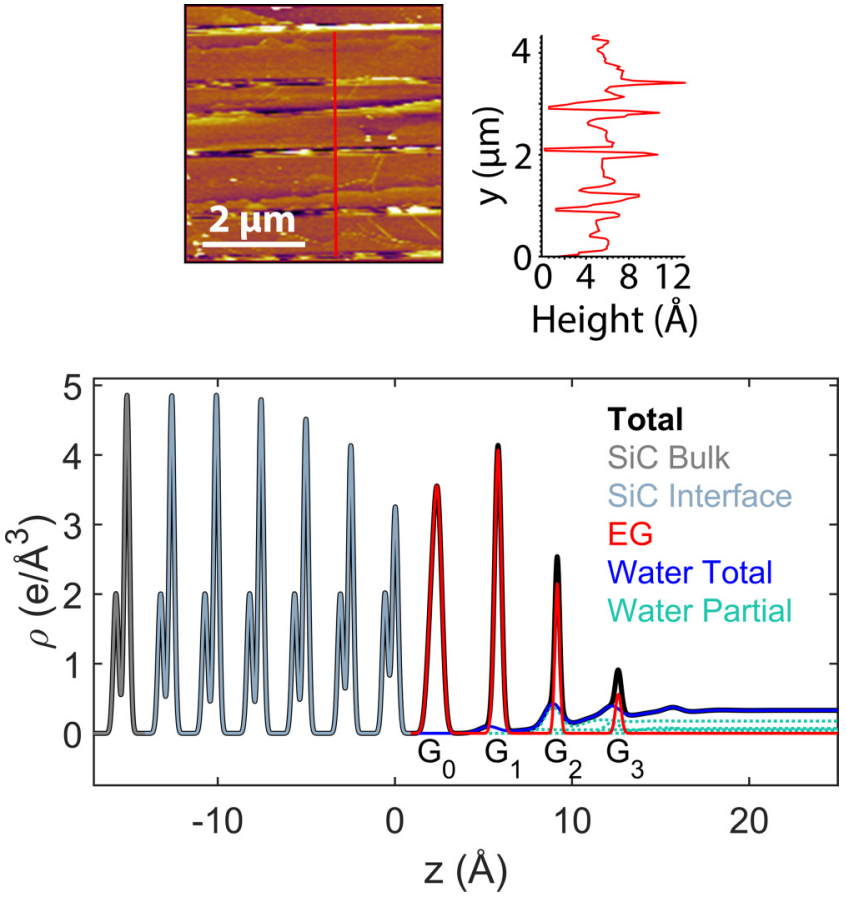

FIG. 12. Top: AFM height image of EG/SiC sample with the line profile (red line) showing the step widths $y$ and heights; sharp spikes in the height profile indicate the step edges. Bottom: resolutionbroadened best-fit electron density profile from the CTR data with $\chi^{2}=1.6$ [Fig. 2(a) in main text], including SiC substrate results and the partial water layers adsorbed on each exposed surface; $u_{\text {broad }}=$ $\left(u_{i}^{2}+u_{\mathrm{res}}^{2}\right)^{1 / 2}$ where $u_{\mathrm{res}}=0.55 / Q_{\max }$.

reference point against which to evaluate the XR best-fit structure (Figs. 4(a), 4(b) and 12, bottom].

The optimized XR model parameters $(z, u$, and $\Theta)$ for each atomic layer are summarized in Table I, and the complete bestfit interface structure of deionized water (DIW) on $\mathrm{EG} / \mathrm{SiC}$ is shown in Fig. 12. We partially constrained the surface $\mathrm{SiC}$ and $\mathrm{G}_{0}$ parameters in the XR analysis based on the work of Emery et al. [44]. That study combined the chemical and structural sensitivity of x-ray photoelectron spectroscopy (XPS), x-ray standing waves (XSW), and XR to rigorously characterize the structure of the air/EG/SiC interface and address longstanding debates about the buffer layer, $\mathrm{G}_{0}$, between $\mathrm{SiC}$ and epitaxial graphene. The XPS/XSW measurements were consistent with a carbon-rich buffer layer composed of two chemically distinct and partially overlapping layers, S1 with $\mathrm{sp}^{2}$ hybridization and $\mathrm{S} 2$ with $\mathrm{sp}^{3}$ hybridization, and bonded to $\mathrm{Si}$ atoms of the substrate [66-70]. The results ruled out a proposed Si adatom model [43,71]. Although Emery et al. found that the $\mathrm{EG} / \mathrm{SiC}$ interface was largely identical across all samples studied (grown in ultrahigh vacuum (UHV) or in an $\mathrm{Ar}$ atmosphere with variable graphene coverage), we allowed for the structure parameters in this work to vary up to $10 \%$ from their results. In general, our results are in agreement with those previously reported.

\section{a. $\mathbf{G}_{0}$ buffer layer}

We identified a $\mathrm{G}_{0}$ layer with a mean height of $2.31 \pm$ $0.02 \AA$ above the SiC surface and FWHM of approximately 
TABLE I. XR best-fit results with uncertainties on the last significant figures in parentheses. Values without uncertainties were fixed during analysis.

\begin{tabular}{|c|c|c|c|}
\hline Layer & $z(\AA)$ & $u(\AA)$ & $\Theta\left(A_{\mathrm{UC}}^{-1}\right)$ \\
\hline \multicolumn{4}{|c|}{$\mathrm{SiC}$} \\
\hline $\mathrm{C}$ & $-13.226(5)$ & 0.0922 & 1 \\
\hline $\mathrm{Si}$ & $-12.598(2)$ & 0.0837 & 1 \\
\hline $\mathrm{C}$ & $-10.723(7)$ & 0.0922 & 1 \\
\hline $\mathrm{Si}$ & $-10.076(3)$ & 0.0837 & 1 \\
\hline $\mathrm{C}$ & $-8.204(15)$ & 0.0922 & 1 \\
\hline $\mathrm{Si}$ & $-7.557(5)$ & 0.0837 & $0.988(8)$ \\
\hline $\mathrm{C}$ & $-5.662(17)$ & 0.0922 & 1 \\
\hline $\mathrm{Si}$ & $-5.026(5)$ & 0.0837 & $0.930(21)$ \\
\hline $\mathrm{C}$ & $-3.080(22)$ & 0.0922 & 1 \\
\hline $\mathrm{Si}$ & $-2.488(10)$ & 0.0837 & $0.850(34)$ \\
\hline $\mathrm{C}$ & $-0.555(73)$ & 0.0922 & $1(1)^{\mathrm{a}}$ \\
\hline $\mathrm{Si}$ & $0.019(15)$ & $0.11(13)^{\mathrm{a}}$ & $0.748(43)$ \\
\hline \multicolumn{4}{|c|}{ Graphene } \\
\hline $\mathrm{S} 1\left(\mathrm{G}_{0}\right)$ & $2.41(3)$ & $0.224(25)$ & $2.86(11)$ \\
\hline $\mathrm{S} 2\left(\mathrm{G}_{0}\right)$ & $1.99(5)$ & $0.19(3)$ & $0.86(44)^{\mathrm{a}}$ \\
\hline $\mathrm{G}_{1}$ & $5.82(4)$ & $0.154(22)$ & $2.66(12)$ \\
\hline $\mathrm{G}_{2}$ & $9.16(7)$ & $0.07(27)^{\mathrm{a}}$ & $0.98(18)$ \\
\hline $\mathrm{G}_{3}$ & $12.6(1)$ & $0.12(71)^{\mathrm{a}}$ & $0.32(3)$ \\
\hline \multicolumn{4}{|c|}{ Water } \\
\hline $\mathrm{H}_{2} \mathrm{O}$ & $\begin{array}{c}5.29(34) \\
d_{w} \\
2.42(1.46)^{\mathrm{a}}\end{array}$ & $\begin{aligned} & 0.43(37) \\
= & \bar{u}=1(1)^{\mathrm{a}}\end{aligned}$ & $0.66^{\mathrm{b}}$ \\
\hline
\end{tabular}

${ }^{\text {a Large uncertainties indicate a general insensitivity to these structural }}$ features and magnify uncertainties for other parameters in cases where they covary. As such, these parameters were fixed in the final iterations of the least-squares optimization.

${ }^{\mathrm{b}}$ Calculated from $d_{w}$.

$0.72 \AA$. Similarly, Emery et al. found a mean $\mathrm{G}_{0}$ height of $\approx 2.3 \AA$ with a $\mathrm{G}_{0}$ FWHM of $\approx 0.8-0.9 \AA$. Others have reported $\mathrm{G}_{0}$ heights of $\approx 2.5 \AA[67,71]$. We note that although our XR measurement could not sufficiently resolve the two $G_{0}$ subpeaks $S 1$ and $S 2$, we were unable to obtain a good fit to the data using a single $G_{0}$ peak, likely due to the asymmetry of this layer. The resolution of the XR measurement is given by $r=\pi / Q_{\max } ; Q_{\max }=4.911 \AA^{-1}$, resulting in $r \approx 0.64 \AA$ for the current measurement, which is larger than the S1-to-S2 separation we identified of $0.42 \pm 0.05 \AA$ but smaller than the FWHM of the combined $\mathrm{G}_{0}$ layer. The S1-to-S2 peak separation is in agreement with the 0.35 - $\AA$ spacing reported by Emery et al. We found that the spacing between S1 and S2 was conserved throughout the fitting iterations as both layers moved together with respect to the $\mathrm{SiC}$ surface, lending support for the shape of the buffer layer. Moreover, the distance from S2 to the topmost Si layer of the substrate was $1.97 \pm$ $0.05 \AA$, in agreement with earlier reports $[44,67]$. We find $\mathrm{G}_{0}$ coverage equivalent to $1.18 \pm 0.03 \mathrm{ML}$, in contrast to previous results that identified a layer with essentially the density of graphene $[44,45,70]$. Attempts were made to constrain the $\mathrm{G}_{0}$ density to that of a single graphene layer, but such a density was always found to be inconsistent with the data. The excess carbon density we identified in the $\mathrm{G}_{0}$ layer may account for a surface oxide species not included in our model [44,72-75].
Emery et al. identified via XPS the presence of $\mathrm{SiO}_{\mathrm{x}}$ in several $\mathrm{EG} / \mathrm{SiC}$ samples, which they were also unable to accurately model in the XR data analysis. They estimated the oxygen coverage to vary from $2 \mathrm{O} / \mathrm{nm}^{2}$ in an Ar-grown sample to 6 $\mathrm{O} / \mathrm{nm}^{2}$ in a UHV-grown sample. The excess carbon density of the $\mathrm{G}_{0}$ layer in our Ar-grown sample can equivalently be attributed to an oxygen content of $\approx 5 \mathrm{O} / \mathrm{nm}^{2}$.

\section{b. SiC surface}

We identify a partially depleted SiC surface, consistent with the thermal desorption of $\mathrm{Si}$ during graphene growth [76-78]. We find that $\mathrm{Si}$ was depleted down to the fourth surface layer of the $\mathrm{SiC}$ while the C layers within $\mathrm{SiC}$ were not depleted. The topmost Si layer was displaced away from the bulk and toward the $\mathrm{G}_{0}$ buffer layer. The coverage of the topmost Si layer is consistent with the coverage of the S2 layer of $\mathrm{G}_{0}$ (though we report a large uncertainty on the $\mathrm{S} 2$ coverage; see Table I). We were unable to rigorously quantify the S2 layer coverage due to the large covariance of this parameter with the $\mathrm{S} 1$ coverage as a result of the limited resolution of the XR measurement discussed previously. Therefore, we fixed this parameter after several fitting iterations where it was converging to values consistent with the amount of surface $\mathrm{Si}$ depletion. That is, the coverages of the two layers indicate a one-to-one bonding between dangling $\mathrm{Si}$ atoms and $\mathrm{sp}^{3}$-hybridized carbons, consistent with the proposed growth mechanisms and previous reports [44,76-78].

\section{c. Epitaxial graphene}

We find a graphene film structure that is consistent with the AFM in Fig. 12. Namely, we identify three 2D graphene layers, $G_{1}-G_{3}$, above the $G_{0}$ buffer layer for a total of 1.25 $\pm 0.07 \mathrm{ML}$ of epitaxial graphene. The mean graphene layer spacing is $3.40 \pm 0.07 \AA$, in agreement with the known value [79], and $G_{1}$ is located $3.5 \pm 0.04 \AA$ above $G_{0}$, in agreement with previous reports $[44,69,70]$.

\section{d. Adsorbed water}

The overall water structure results are discussed in the main text, and the best-fit parameter values are given in Table I. Here, we focus on similarities and differences between our results and a those from a previous XR study of the adsorbed water structure on graphene [45] and discuss the implications for the intrinsic interactions of water with graphene.

We find a water height above the free-standing graphene layers $\left(\mathrm{G}_{1}-\mathrm{G}_{3}\right)$ of $\approx 3.1 \AA$, in agreement with the results of Zhou et al. [45]. However, we identify significant differences in the water structure above the $G_{0}$ buffer layer. We find that water adsorbs closer to the buffer layer at $\approx 3 \AA$ above $\mathrm{G}_{0}$, but the height uncertainty overlaps with the water height above free-standing graphene and indicates that the buffer layer is also weakly hydrophobic. We note that although we assumed a single water model above all "graphene" surfaces in our analysis (including $G_{0}$ ), the different height for the buffer layer results from calculating the distance between water and the weighted average position of the S1 and S2 peaks, a condition that was not imposed during the XR data analysis. In contrast, Zhou et al. found that water adsorbs at a height of $\approx 2.33 \AA$ above the buffer layer, indicating a hydrophilic 
character. They report water contact angle (WCA) measurements that support a more hydrophilic $\mathrm{G}_{0}$ and which are equivalent to the WCA on bare SiC [80]. The WCA measurements showed a linearly increasing trend with graphene layer thickness, which may initially suggest that the buffer layer and subsequent graphene layers display wetting transparency on $\mathrm{SiC}$. However, the extent of graphene's wetting transparency is still contested [23,80-82], and it is unclear if the $\mathrm{G}_{0}$ layer with its $\mathrm{sp}^{2}$ and $\mathrm{sp}^{3}$ character would exhibit similar wetting transparency properties. Instead, the evidence presented by Zhou et al. suggest that the $\mathrm{G}_{0}$ water adsorption is more in line with a high concentration of defects on their samples.

Zhou et al. used UHV-grown EG/SiC samples, which are known to possess a greater amount of defects than graphene grown in a furnace in an $\mathrm{Ar}$ atmosphere (as was done for the sample studied in this work), as shown by their AFM images and other reports [83]. Based on the results of Emery et al. wherein UHV-grown and Ar-grown $\mathrm{EG} / \mathrm{SiC}$ were found to have equivalent interface structures [44], which are consistent with our own EG/SiC interface structure, we would not expect the growth methodology to substantially contribute to the differences observed between our and Zhou's $\mathrm{G}_{0}$-water distance. However, the quality of the sample depends on the vacuum level and any pretreatments of the $\mathrm{SiC}$ to remove oxides $[72,73,75,84]$. No pretreatments were reported by Zhou et al. They also reported Raman data with significant $D$ and $D+D^{\prime}$ peaks, which result from edge and other defect states [80,8588]. In fact, it has been shown that the introduction of such defect peaks upon oxygen plasma etching of $\mathrm{EG} / \mathrm{SiC}$ is associated with a decrease in WCA [80]. Finally, Zhou et al. report MD and $a b$ initio MD (AIMD) simulations of defect-free surfaces that predict water heights above $\mathrm{G}_{0}$ consistent with that observed above free-standing graphene and in agreement with our MD results. The AIMD simulations included effects of the $\mathrm{SiC}$ substrate and the corrugation of the buffer layer, but found only an $\approx 0.2 \AA$ decrease in the adsorbed water height above $\mathrm{G}_{0}$ compared to free-standing graphene. Only upon inclusion of Si vacancies and -OH defects were they able to simulate a $\mathrm{G}_{0}$-water height of $2.33 \AA$. We conclude that while we can reasonably expect water to adsorb more closely to the buffer layer than to the subsequent graphene layers as a result of the $\mathrm{SiC}$ substrate and corrugated surface, we expect the effect to be minor in the absence of substantial defects. For a defect-free surface, the water structures above the buffer layer and subsequent graphene layers are very similar and can be described well by a single water model given certain resolution limits of the XR measurement.

\section{Agreement and differences with simulation}

Figure 13 shows adsorbed water profiles isolated from each exposed graphene layer [see Fig. 2(d) in the main text for the total density profile]. Although the XR and MD results generally agree, a noticeable broadening of the first hydration layer is predicted by MD above each partial graphene surface. The width is consistent with the XR result by the third layer $\left(G_{2}\right)$. A graphene slab of four uniform and complete layers produces the same water structure as is shown in Fig. 13 for the layer $\mathrm{G}_{0}$. The origins of these discrepancies should be

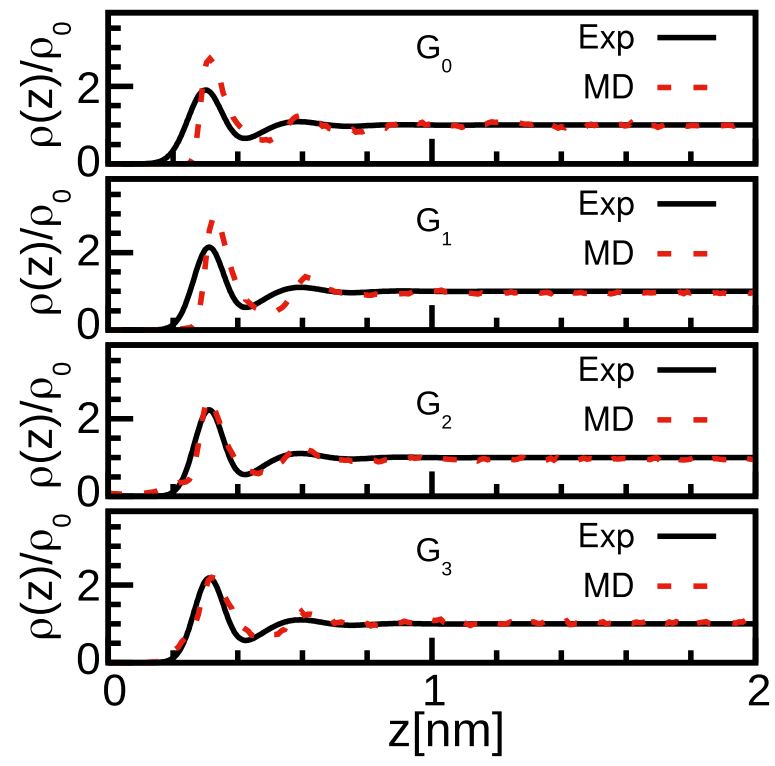

FIG. 13. Density profiles normalized with respect to the bulk water density on top of the layer $\mathrm{G}_{i}(i=0, \ldots, 3)$ from experiments (solid black line) and from MD simulations (dashed red line). The widths of the hydration layers in the experiment are broadened according to Eq. (18).

discussed in the context of the limitations of both the MD and XR approaches:

(1) The $\mathrm{G}_{0}$ layer of the MD simulation is modeled as a $2 \mathrm{D}$ graphene layer due to the absence of a $\mathrm{SiC}$ substrate. However, the partial $\mathrm{sp}^{2}$ and $\mathrm{sp}^{3}$ character of $\mathrm{G}_{0}$ on $\mathrm{SiC}$ alters the band structure [89] and may affect the buffer layer's interaction with water. Indeed, this is consistent with our XR results and previous AIMD predictions [45], though the effect is weak. Previous MD simulations of unsupported graphene sheets suggest via water contact angle calculations that the graphene hydrophobicity decreases with increasing layer thickness [82] and becomes graphite-like by the third layer [90]. This agrees with our MD simulations wherein the adsorbed water peak height is conserved, but the broadening reveals a small increase in the number of water molecules that are able to adsorb closer to the graphene sheet. (2) The XR-derived result is limited by the complexity of the XR analysis model, as described in detail above. We used a single intrinsic water model for each graphene surface despite evidence that on $\mathrm{SiC}$ thicker graphene regions are more hydrophobic than thinner regions [91]. We note that this is the opposite of MD predictions, suggesting that while the effect of the SiC substrate and corrugated buffer layer are relatively weak in magnitude, they may significantly affect the chemistry of the surface. Accounting for different hydrophobicity would introduce additional parameters to an already complex model and is unnecessary given that the changes are small. Using a single water model essentially gives an optimized structure that captures the average behavior of water adsorption on an imperfect graphene surface. In addition, the nonlinear least-squares fitting of the data can produce multiple equally viable structures (i.e., with equivalently good $\chi^{2}$ ). This point emphasizes the importance of including evidence from other experimental methodologies 
such as XPS/XSW [44] to refine and constrain models. Even with these caveats, the qualitative agreement between the XR and MD structures, paired with the high-confidence in the XR result (given a $\chi^{2}$ of 1.6 where a perfect fit would have a $\chi^{2}$ of 1 ), provides a consistent picture of water adsorption on graphene.
[1] P. G. Debenedetti and M. L. Klein, Chemical physics of water, Proc. Natl. Acad. Sci. USA 114, 13325 (2017).

[2] Y. Gogotsi, Moving ions confined between graphene sheets, Nat. Nanotech. 13, 625 (2018).

[3] B. Panganiban, B. Qiao, T. Jiang, C. DelRe, M. M. Obadia, T. D. Nguyen, A. A. A. Smith, A. Hall, I. Sit, M. G. Crosby, P. B. Dennis, E. Drockenmuller, M. Olvera de la Cruz, and T. Xu, Random heteropolymers preserve protein function in foreign environments, Science 359, 1239 (2018).

[4] C. Krekeler, F. Dommert, J. Schmidt, Y. Y. Zhao, C. Holm, R. Berger, and L. Delle Site, Electrostatic properties of liquid 1,3dimethylimidazolium chloride: Role of local polarization and effect of the bulk, Phys. Chem. Chem. Phys. 12, 1817 (2010).

[5] N. Giovambattista, P. J. Rossky, and P. G. Debenedetti, Phase Transitions Induced by Nanoconfinement in Liquid Water, Phys. Rev. Lett. 102, 050603 (2009).

[6] M. Neek-Amal, F. M. Peeters, I. V. Grigorieva, and A. K. Geim, Commensurability effects in viscosity of nanoconfined water, ACS Nano 10, 3685 (2016).

[7] K. Wu, Z. Chen, J. Li, X. Li, J. Xu, and X. Dong, Wettability effect on nanoconfined water flow, Proc. Natl. Acad. Sci. USA 114, 3358 (2017).

[8] K.-i. Otake, K. Otsubo, T. Komatsu, S. Dekura, J. M. Taylor, R. Ikeda, K. Sugimoto, A. Fujiwara, C.-P. Chou, A. W. Sakti, Y. Nishimura, H. Nakai, and H. Kitagawa, Confined watermediated high proton conduction in hydrophobic channel of a synthetic nanotube, Nat. Commun. 11, 843 (2020).

[9] M. F. Toney, J. N. Howard, J. Richer, G. L. Borges, J. G. Gordon, O. R. Melroy, D. G. Wiesler, D. Yee, and L. B. Sorensen, Voltage-dependent ordering of water molecules at an electrode-electrolyte interface, Nature (London) 368, 444 (1994).

[10] S. Chen, Y. Itoh, T. Masuda, S. Shimizu, J. Zhao, J. Ma, S. Nakamura, K. Okuro, H. Noguchi, K. Uosaki, and T. Aida, Subnanoscale hydrophobic modulation of salt bridges in aqueous media, Science 348, 555 (2015).

[11] O. Teschke and E. F. de Souza, Electrostatic response of hydrophobic surface measured by atomic force microscopy, Appl. Phys. Lett. 82, 1126 (2003).

[12] L. Fumagalli, A. Esfandiar, R. Fabregas, S. Hu, P. Ares, A. Janardanan, Q. Yang, B. Radha, T. Taniguchi, K. Watanabe, G. Gomila, K. S. Novoselov, and A. K. Geim, Anomalously low dielectric constant of confined water, Science 360, 1339 (2018).

[13] V. Ballenegger and J.-P. Hansen, Dielectric permittivity profiles of confined polar fluids, J. Chem. Phys. 122, 114711 (2005).

[14] H. A. Stern and S. E. Feller, Calculation of the dielectric permittivity profile for a nonuniform system: Application to a lipid bilayer simulation, J. Chem. Phys. 118, 3401 (2003).

[15] D. J. Bonthuis, S. Gekle, and R. R. Netz, Profile of the static permittivity tensor of water at interfaces: Consequences for capacitance, hydration interaction, and ion adsorption, Langmuir 28, 7679 (2012).
[16] H. Jalali, H. Ghorbanfekr, I. Hamid, M. Neek-Amal, R. Rashidi, and F. M. Peeters, Out-of-plane permittivity of confined water, Phys. Rev. E 102, 022803 (2020).

[17] Y. Jing, V. Jadhao, J. W. Zwanikken, and M. Olvera de la Cruz, Ionic structure in liquids confined by dielectric interfaces, J. Chem. Phys. 143, 194508 (2015).

[18] C. D. Williams, J. Dix, A. Troisi, and P. Carbone, Effective polarization in pairwise potentials at the graphene-electrolyte interface, J. Phys. Chem. Lett. 8, 703 (2017).

[19] D. J. Cole, P. K. Ang, and K. P. Loh, Ion adsorption at the graphene/electrolyte interface, J. Phys. Chem. Lett. 2, 1799 (2011).

[20] C. Zhan, M. R. Cerón, S. A. Hawks, M. Otani, B. C. Wood, T. A. Pham, M. Stadermann, and P. G. Campbell, Specific ion effects at graphitic interfaces, Nat. Commun. 10, 1 (2019).

[21] C. Zhan, F. Aydin, E. Schwegler, A. Noy, and T. A. Pham, Understanding cation selectivity in carbon nanopores with hybrid first-principles/continuum simulations: Implications for water desalination and separation technologies, ACS Appl. Nano Mat. 3, 9740 (2020).

[22] A. K. Geim and K. S. Novoselov, The rise of graphene, Nat. Mater. 6, 183 (2007).

[23] J. Rafiee, X. Mi, H. Gullapalli, A. V. Thomas, F. Yavari, Y. Shi, P. M. Ajayan, and N. A. Koratkar, Wetting transparency of graphene, Nat. Mater. 11, 217 (2012).

[24] D. Cohen-Tanugi and J. C. Grossman, Water desalination across nanoporous graphene, Nano Lett. 12, 3602 (2012).

[25] S. P. Surwade, S. N. Smirnov, I. V. Vlassiouk, R. R. Unocic, G. M. Veith, S. Dai, and S. M. Mahurin, Water desalination using nanoporous single-layer graphene, Nat. Nanotech. 10, 459 (2015).

[26] R. Raccichini, A. Varzi, S. Passerini, and B. Scrosati, The role of graphene for electrochemical energy storage, Nat. Mater. 14, 271 (2015).

[27] P. Dhiman, F. Yavari, X. Mi, H. Gullapalli, Y. Shi, P. M. Ajayan, and N. Koratkar, Harvesting energy from water flow over graphene, Nano Lett. 11, 3123 (2011).

[28] S. Garaj, W. Hubbard, A. Reina, J. Kong, D. Branton, and J. Golovchenko, Graphene as a subnanometre trans-electrode membrane, Nature (London) 467, 190 (2010).

[29] K. A. Perrine, K. M. Parry, A. C. Stern, M. H. C. Van Spyk, M. J. Makowski, J. A. Freites, B. Winter, D. J. Tobias, and J. C. Hemminger, Specific cation effects at aqueous solutionvapor interfaces: Surfactant-like behavior of $\mathrm{Li}+$ revealed by experiments and simulations, Proc. Natl. Acad. Sci. USA 114, 13363 (2017).

[30] D. Horinek, A. Herz, L. Vrbka, F. Sedlmeier, S. I. Mamatkulov, and R. R. Netz, Specific ion adsorption at the air/water interface: The role of hydrophobic solvation, Chem. Phys. Lett. 479, 173 (2009).

[31] D. L. McCaffrey, S. C. Nguyen, S. J. Cox, H. Weller, A. P. Alivisatos, P. L. Geissler, and R. J. Saykally, Mechanism of ion 
adsorption to aqueous interfaces: Graphene/water vs. air/water, Proc. Natl. Acad. Sci. USA 114, 13369 (2017).

[32] H. J. C. Berendsen, J. R. Grigera, and T. P. Straatsma, The missing term in effective pair potentials, J. Phys. Chem. 91, 6269 (1987).

[33] W. L. Jorgensen, D. S. Maxwell, and J. Tirado-Rives, Development and testing of the OPLS all-atom force field on conformational energetics and properties of organic liquids, J. Am. Chem. Soc. 118, 11225 (1996).

[34] I.-C. Yeh and M. L. Berkowitz, Ewald summation for systems with slab geometry, J. Chem. Phys. 111, 3155 (1999).

[35] J. D. Jackson, Classical Electrodynamics, 3rd ed. (Wiley, New York, 1999).

[36] G. Torrie and J. Valleau, Nonphysical sampling distributions in monte carlo free-energy estimation: Umbrella sampling, J. Comp. Phys. 23, 187 (1977).

[37] J. Kästner, Umbrella sampling, WIREs Comput. Mol. Sci. 1, 932 (2011)

[38] B. Hess, C. Kuttner, D. van der Spoel, and E. Lindahl, GROMACS 4: Algorithms for highly efficient, load-balanced, and scalable molecular simulation, J. Chem. Theory Comput. 4, 435 (2008).

[39] T. D. Nguyen, H. Li, D. Bagchi, F. J. Solis, and M. Olvera de la Cruz, Incorporating surface polarization effects into large-scale coarse-grained molecular dynamics simulation, Comput. Phys. Commun. 241, 80 (2019).

[40] J. W. Zwanikken and M. Olvera de la Cruz, Tunable soft structure in charged fluids confined by dielectric interfaces, Proc. Natl. Acad. Sci. USA 110, 5301 (2013).

[41] G. Renaud, Oxide surfaces and metal/oxide interfaces studied by grazing incidence x-ray scattering, Surf. Sci. Rep. 32, 5 (1998).

[42] P. Fenter and S. S. Lee, Hydration layer structure at solid-water interfaces, MRS Bull. 39, 1056 (2014).

[43] J. Hass, J. E. Millán-Otoya, P. N. First, and E. H. Conrad, Interface structure of epitaxial graphene grown on $4 \mathrm{H}-\mathrm{SiC}(0001)$, Phys. Rev. B 78, 205424 (2008).

[44] J. D. Emery, B. Detlefs, H. J. Karmel, L. O. Nyakiti, D. K. Gaskill, M. C. Hersam, J. Zegenhagen, and M. J. Bedzyk, Chemically Resolved Interface Structure of Epitaxial Graphene on SiC(0001), Phys. Rev. Lett. 111, 215501 (2013).

[45] H. Zhou, P. Ganesh, V. Presser, M. C. F. Wander, P. Fenter, P. R. C. Kent, D. E. Jiang, A. A. Chialvo, J. McDonough, K. L. Shuford, and Y. Gogotsi, Understanding controls on interfacial wetting at epitaxial graphene: Experiment and theory, Phys. Rev. B 85, 035406 (2012).

[46] K. V. Emtsev, A. Bostwick, K. Horn, J. Jobst, G. L. Kellogg, L. Ley, J. L. McChesney, T. Ohta, S. A. Reshanov, J. Röhrl, E. Rotenberg, A. K. Schmid, D. Waldmann, H. B. Weber, and T. Seyller, Towards wafer-size graphene layers by atmospheric pressure graphitization of silicon carbide, Nat. Mater. 8, 203 (2009).

[47] W. A. de Heer, C. Berger, M. Ruan, M. Sprinkle, X. Li, Y. Hu, B. Zhang, J. Hankinson, and E. Conrad, Large area and structured epitaxial graphene produced by confinement controlled sublimation of silicon carbide, Proc. Natl. Acad. Sci. USA 108, 16900 (2011).

[48] P. J. Brown, A. G. Fox, E. N. Maslen, M. A. O’Keefe, and B. T. M. Willis, Intensity of diffracted intensities, in International Tables for Crystallography, edited by E. Prince
(International Union of Crystallography, Springer, Dordrecht, 2006), Vol. C, Chap. 6.1, pp. 554-595.

[49] G. C. Capitani, S. Di Pierro, and G. Tempesta, The 6H-SiC structure model: Further refinement from SCXRD data from a terrestrial moissanite, Am. Mineral. 92, 403 (2007).

[50] O. M. Magnussen, B. M. Ocko, M. J. Regan, K. Penanen, P. S. Pershan, and M. Deutsch, X-Ray Reflectivity Measurements of Surface Layering in Liquid Mercury, Phys. Rev. Lett. 74, 4444 (1995).

[51] A. P. dos Santos and Y. Levin, Electrolytes between dielectric charged surfaces: Simulations and theory, J. Chem. Phys. 142, 194104 (2015).

[52] R. Messina, Effect of image forces on polyelectrolyte adsorption at a charged surface, Phys. Rev. E 70, 051802 (2004).

[53] A. Uysal, M. Chu, B. Stripe, A. Timalsina, S. Chattopadhyay, C. M. Schlepütz, T. J. Marks, and P. Dutta, What x rays can tell us about the interfacial profile of water near hydrophobic surfaces, Phys. Rev. B 88, 035431 (2013).

[54] M. Mezger, F. Sedlmeier, D. Horinek, H. Reichert, D. Pontoni, and $\mathrm{H}$. Dosch, On the origin of the hydrophobic water gap: An X-ray reflectivity and MD simulation study, J. Am. Chem. Soc. 132, 6735 (2010).

[55] F. Jiménez-Ángeles and A. Firoozabadi, Hydrophobic hydration and the effect of $\mathrm{NaCl}$ salt in the adsorption of hydrocarbons and surfactants on clathrate hydrates, ACS Central Sci. 4, 820 (2018).

[56] C. Zhang, F. Gygi, and G. Galli, Strongly anisotropic dielectric relaxation of water at the nanoscale, J. Phys. Chem. Lett. 4, 2477 (2013).

[57] K. Ji, J. Han, A. Hirata, T. Fujita, Y. Shen, S. Ning, P. Liu, H. Kashani, Y. Tian, Y. Ito et al., Lithium intercalation into bilayer graphene, Nat. Commun. 10, 275 (2019).

[58] B. Qiao, F. Jiménez-Ángeles, T. D. Nguyen, and M. Olvera de la Cruz, Water follows polar and nonpolar protein surface domains, Proc. Natl. Acad. Sci. USA 116, 19274 (2019).

[59] A. P. dos Santos and Y. Levin, Ions at the water-oil interface: Interfacial tension of electrolyte solutions, Langmuir 28, 1304 (2012).

[60] G. Lamoureux, E. Harder, I. V. Vorobyov, B. Roux, and A. D. MacKerell, A polarizable model of water for molecular dynamics simulations of biomolecules, Chem. Phys. Lett. 418, 245 (2006).

[61] H. Yu, T. W. Whitfield, E. Harder, G. Lamoureux, I. Vorobyov, V. M. Anisimov, A. D. MacKerell, and B. Roux, Simulating monovalent and divalent ions in aqueous solution using a Drude polarizable force field, J. Chem. Theory Comput. 6, 774 (2010).

[62] R. P. Misra and D. Blankschtein, Insights on the role of manybody polarization effects in the wetting of graphitic surfaces by water, J. Phys. Chem. C 121, 28166 (2017).

[63] G. Lamoureux and B. Roux, Modeling induced polarization with classical Drude oscillators: Theory and molecular dynamics simulation algorithm, J. Chem. Phys. 119, 3025 (2003).

[64] T. A. Ho and A. Striolo, Polarizability effects in molecular dynamics simulations of the graphene-water interface, J. Chem. Phys. 138, 054117 (2013).

[65] I. K. Robinson, Crystal truncation rods and surface roughness, Phys. Rev. B 33, 3830 (1986).

[66] K. V. Emtsev, T. Seyller, F. Speck, L. Ley, P. Stojanov, J. D. Riley, and R. C. G. Leckey, Initial stages of the 
graphite-SiC(0001) interface formation studied by photoelectron spectroscopy, Mater. Sci. Forum, 556-557, 525 (2007).

[67] A. Mattausch and O. Pankratov, Ab initio Study of Graphene on SiC, Phys. Rev. Lett. 99, 076802 (2007).

[68] F. Varchon, R. Feng, J. Hass, X. Li, B. N. Nguyen, C. Naud, P. Mallet, J.-Y. Veuillen, C. Berger, E. H. Conrad, and L. Magaud, Electronic Structure of Epitaxial Graphene Layers on SiC: Effect of the Substrate, Phys. Rev. Lett. 99, 126805 (2007).

[69] F. Varchon, P. Mallet, J.-Y. Veuillen, and L. Magaud, Ripples in epitaxial graphene on the Si-terminated $\mathrm{SiC}(0001)$ surface, Phys. Rev. B 77, 235412 (2008).

[70] S. Kim, J. Ihm, H. J. Choi, and Y.-W. Son, Origin of Anomalous Electronic Structures of Epitaxial Graphene on Silicon Carbide, Phys. Rev. Lett. 100, 176802 (2008).

[71] G. M. Rutter, N. P. Guisinger, J. N. Crain, E. A. A. Jarvis, M. D. Stiles, T. Li, P. N. First, and J. A. Stroscio, Imaging the interface of epitaxial graphene with silicon carbide via scanning tunneling microscopy, Phys. Rev. B 76, 235416 (2007).

[72] J. Bernhardt, J. Schardt, U. Starke, and K. Heinz, Epitaxially ideal oxide-semiconductor interfaces: Silicate adlayers on hexagonal (0001) and $\left(0001^{-}\right) \mathrm{SiC}$ surfaces, Appl. Phys. Lett. 74, 1084 (1999).

[73] Y. Hoshino, T. Nishimura, T. Yoneda, K. Ogawa, H. Namba, and $\mathrm{Y}$. Kido, Initial oxidation of $6 \mathrm{H}-\mathrm{SiC}(0001)-3 \times 3$ surface studied by ion scattering combined with photoemission induced by synchrotron-radiation-light, Surf. Sci. 505, 234 (2002).

[74] F. Amy, H. Enriquez, P. Soukiassian, P.-F. Storino, Y. J. Chabal, A. J. Mayne, G. Dujardin, Y. K. Hwu, and C. Brylinski, Atomic Scale Oxidation of a Complex System: $\mathrm{O}_{2} / \alpha-\operatorname{SiC}(0001)-(3 \times$ 3), Phys. Rev. Lett. 86, 4342 (2001).

[75] F. Amy, P. Soukiassian, Y. K. Hwu, and C. Brylinski, Sirich $6 h$ - and $4 h-\mathrm{SiC}(0001) 3 \times 3$ surface oxidation and initial $\mathrm{SiO}_{2} / \mathrm{SiC}$ interface formation from 25 to $650^{\circ} \mathrm{C}$, Phys. Rev. B 65, 165323 (2002).

[76] A. V. Bommel, J. Crombeen, and A. V. Tooren, Leed and auger electron observations of the $\mathrm{SiC}(0001)$ surface, Surf. Sci. 48, 463 (1975).

[77] I. Forbeaux, J.-M. Themlin, and J.-M. Debever, Heteroepitaxial graphite on $6 h$ - $\mathrm{SiC}(0001)$ : Interface formation through conduction-band electronic structure, Phys. Rev. B 58, 16396 (1998).
[78] H. Huang, W. Chen, S. Chen, and A. T. S. Wee, Bottom-up growth of epitaxial graphene on $6 \mathrm{H}-\mathrm{SiC}(0001)$, ACS Nano 2, 2513 (2008).

[79] Y. Baskin and L. Meyer, Lattice constants of graphite at low temperatures, Phys. Rev. 100, 544 (1955).

[80] Y. J. Shin, Y. Wang, H. Huang, G. Kalon, A. T. S. Wee, Z. Shen, C. S. Bhatia, and H. Yang, Surface-energy engineering of graphene, Langmuir 26, 3798 (2010).

[81] R. Raj, S. C. Maroo, and E. N. Wang, Wettability of graphene, Nano Lett. 13, 1509 (2013).

[82] C.-J. Shih, Q. H. Wang, S. Lin, K.-C. Park, Z. Jin, M. S. Strano, and D. Blankschtein, Breakdown in the Wetting Transparency of Graphene, Phys. Rev. Lett. 109, 176101 (2012).

[83] K. V. Emtsev, F. Speck, T. Seyller, L. Ley, and J. D. Riley, Interaction, growth, and ordering of epitaxial graphene on SiC 0001 surfaces: A comparative photoelectron spectroscopy study, Phys. Rev. B 77, 155303 (2008).

[84] T. Seyller, Electronic properties of SiC surfaces and interfaces: Some fundamental and technological aspects, Appl. Phys. A 85, 371 (2006).

[85] F. Tuinstra and J. L. Koenig, Raman spectrum of graphite, J. Chem. Phys. 53, 1126 (1970).

[86] A. C. Ferrari and D. M. Basko, Raman spectroscopy as a versatile tool for studying the properties of graphene, Nat. Nanotechnol. 8, 235 (2013).

[87] Z. H. Ni, W. Chen, X. F. Fan, J. L. Kuo, T. Yu, A. T. S. Wee, and Z. X. Shen, Raman spectroscopy of epitaxial graphene on a SiC substrate, Phys. Rev. B 77, 115416 (2008).

[88] C. Casiraghi, A. Hartschuh, H. Qian, S. Piscanec, C. Georgi, A. Fasoli, K. S. Novoselov, D. M. Basko, and A. C. Ferrari, Raman spectroscopy of graphene edges, Nano Lett. 9, 1433 (2009).

[89] T. Ohta, A. Bostwick, J. L. McChesney, T. Seyller, K. Horn, and E. Rotenberg, Interlayer Interaction and Electronic Screening in Multilayer Graphene Investigated with Angle-Resolved Photoemission Spectroscopy, Phys. Rev. Lett. 98, 206802 (2007).

[90] F. Taherian, V. Marcon, N. F. A. van der Vegt, and F. Leroy, What is the contact angle of water on graphene? Langmuir 29, 1457 (2013).

[91] M. Munz, C. E. Giusca, R. L. Myers-Ward, D. K. Gaskill, and O. Kazakova, Thickness-dependent hydrophobicity of epitaxial graphene, ACS Nano 9, 8401 (2015). 